\title{
FLOW EQUIVALENCE OF SOFIC SHIFTS
}

\author{
MIKE BOYLE, TOKE MEIER CARLSEN, AND SØREN EILERS
}

\begin{abstract}
We classify certain sofic shifts (the irreducible Point Extension Type, or PET, sofic shifts) up to flow equivalence, using invariants of the canonical Fischer cover. There are two main ingredients.

(1) An extension theorem, for extending flow equivalences of subshifts to flow equivalent irreducible shifts of finite type which contain them.

(2) The classification of certain constant to one maps from SFTs via algebraic invariants of associated $G$-SFTs.
\end{abstract}

\section{Contents}

1. Introduction

2. Flow equivalence

3. Multiplicity sets of canonical covers

4. The extension theorem

5. The reduction theorem for AFT shifts

6. $\quad N$-point extensions and $G$-SFTs

7. PET sofic shifts

8. Algorithms for PET sofic shifts

References

\section{INTRODUCTION}

Suppose for $i=1,2$ that $\sigma_{i}: X_{i} \rightarrow X_{i}$ is a homeomorphism of a compact metric space. Then $\sigma_{1}$ and $\sigma_{2}$ are flow equivalent if they are topologically conjugate to return maps to cross sections of a common flow. Equivalently, there is a homeomorphism between their mapping tori which respects orbits and orientation of the suspension flows. Return maps to cross sections of flows arose historically in the study of differential equations, and were abstracted to topological dynamics on compact metric spaces [36]; they were later absorbed into a theory of cocycles for general group actions [26].

For zero-dimensional systems, flow equivalence is a multifaceted relation. Flow equivalence of shifts of finite type turns out to be a significant feature of an overall algebraic framework for analyzing the 
topological dynamics of SFTs and free $G$-SFTs (SFTs with a continuous shift commuting free action by a finite group $G$ ) (see [18] and its references). Flow equivalence is a significant ingredient in the ongoing interplay between symbolic dynamics and $C^{*}$-algebras (see [33] and its many references). Especially, the classification of shifts of finite type up to flow equivalence (by Franks [20] in the irreducible case and by Huang [7, 11, 23] in general) was a critical ingredient in the classification of Cuntz-Krieger algebras of real rank zero up to stable isomorphism [38, 39]. Flow equivalence of some subshift classes has been studied with category theory, semigroup theory and sophisticated formal language analysis [19, 30]. The Barge-Diamond topological classification [3] of one-dimensional substitution tiling spaces amounts to the classification of the associated substitution subshifts up to flow equivalence, and a recent paper by Johansen ([24]) addresses the case of beta-shifts.

In this paper, we study flow equivalence of sofic shifts (the class of subshifts most closely generalizing the shifts of finite type), and especially the almost finite type (AFT) class of Marcus [32, by means of their canonical covers. There is a natural notion of flow equivalence of such covers, which turns out to be a complete invariant of flow equivalence of the associated sofic shifts. We use the Fischer covers covers to classify a large (but far from general) class of AFT shifts up to flow equivalence. There are two main ingredients for this.

First, relying on [14] we prove an extension theorem which lets us extend a flow equivalence of a subflow to a flow equivalence of mapping tori of flow equivalent irreducible SFTs. With the known classification of irreducible SFTs up to flow equivalence, this reduces the flow equivalence classification of AFT shifts to the problem of classifying the restrictions of their Fischer covers to their multiplicity shifts.

Next, we introduce a new class of sofic shifts, contained in the class of AFT shifts: the "point extension type" (PET) sofic shifts. A sofic shift $Y$ is PET if for its Fischer cover $\pi$ and each $k>1$, the set $\mathrm{M}_{k}(\pi):=\left\{y \in Y:\left|\pi^{-1}(y)\right|=k\right\}$ is closed. For these shifts, drawing further on work of Adler, Kitchens and Marcus [1 (following Rudolph [40]), we reduce the analysis of these restricted covers to the problem of classifying associated G-SFTs up to equivariant flow equivalence. We solve that problem in a separate paper [9]. The complete algebraic invariants are manageable for constructions and for analyzing some classes. We do not know if there is a decision procedure for determining $G$-flow equivalence in general, but many cases can be decided (see Remark 7.13 and [17]).

There is a natural notion of shift equivalence for sofic shifts [13, 22] which by work of Kim and Roush is decidable [27. The algebraic framework for that work should also be appropriate for studying flow equivalence of sofic shifts and its decidability. Ultimately, the flow 
equivalence of sofic shifts may be understood from both viewpoints, each with its advantages.

The paper has the following structure. Sections 2 and 3 are background. Sections 4 and 5 have our main result (the Extension Theorem) and its main application (the Reduction Theorem). The Reduction Theorem is applied in Section 5 to classify the near Markov shifts up to flow equivalence and establish that such shifts spaces are always flow equivalent to their time-reversals. In Section 6, following Adler, Kitchens and Marcus [1, 2] we explain how topological conjugacy of certain constant to one maps is equivalent to the topological conjugacy of certain associated $G$-SFTs. In Section 8, we apply this to classify PET (point extension type) sofic shifts up to flow equivalence. Finally, in Section 8, we give procedures to determine whether a sofic shift is PET and to compute matrices over $\mathbb{Z}_{+} G$ from which the algebraic invariants are defined.

This work was supported by the Danish National Research Foundation through the Centre for Symmetry and Deformation (DNRF92), and by VILLUM FONDEN through the network for Experimental Mathematics in Number Theory, Operator Algebras, and Topology.

\section{Flow EQUivalence}

We give a detailed treatment of flow equivalence for subshifts in [8. Here we give just some basic properties needed for this paper. The reader is referred to [31] for further background on basic symbolic dynamics.

For a finite alphabet $\mathfrak{a}$ we will by $\sigma_{\mathfrak{a}}$ (or just $\sigma$ when the alphabet has been fixed) denote the shift map on $\mathfrak{a}^{\mathbb{Z}}$. By a shift space $X$, we mean a closed shift invariant subset $X$ of some $\mathfrak{a}^{\mathbb{Z}}$; we use $X$ also to represent the dynamical system which is the restriction of the shift to $X$. For a shift space $X$ we will by $\mathfrak{a}(X)$ denote the alphabet of $X$.

For a shift space $X$, the mapping torus is the quotient space

$$
\mathrm{S} X=X \times \mathbb{R} / \sim
$$

with

$$
(x, t) \sim(y, s) \Longleftrightarrow t-s \in \mathbb{Z} \wedge y=\sigma^{t-s}(x) .
$$

Note that $\mathrm{S} X$ comes equipped with an action of $\mathbb{R}$ :

$$
\gamma_{t_{0}}([x, t])=\left[x, t+t_{0}\right]
$$

We say that two shift spaces $X$ and $Y$ are flow equivalent if there exists a homeomorphism $\psi: \mathrm{S} X \rightarrow \mathrm{S} Y$ which is orientation preserving in the sense that for each $x \in X$ there is an increasing function $f_{x}: \mathbb{R} \rightarrow$ $\mathbb{R}$ with the property

$$
\psi\left(\gamma_{t}(x)\right)=\gamma_{f_{x}(t)}(\psi(x))
$$


We call the map $\psi$ an equivalence of flows, or a flow equivalence, and denote the flow equivalence class of $X$ by $[[X]]_{F E}$. A key notion for us is that of a cross section:

Definition 2.1. A subset $R \subset \mathrm{S} X$ is called a cross section when the map $s: \mathbb{R} \times R \rightarrow \mathrm{S} X$ given by

$$
s(t, x)=\gamma_{t}(x)
$$

is a surjective local homeomorphism.

If $R \subset \mathrm{S} X$ is cross section, then there is a well defined return map $\rho: R \rightarrow R$ such that $\rho(x)=\gamma_{t}(x)$ where $t=\min \left\{t>0: \gamma_{t}(x) \in R\right\}$. The dynamical system $(R, \rho)$ is conjugate to a shift space $X_{R}$, and $\mathrm{S} X_{R}$ can in a natural way be identified with $S X$. Note that $X \times\{0\} / \sim$ is always a cross section for $\mathrm{S} X$ and that $(X \times\{0\} / \sim, \rho)$ is conjugate to $(X, \sigma)$. Throughout this paper, we will identify $(X, \sigma)$ with $(X \times\{0\} / \sim$ $, \rho)$.

Definition 2.2. When $F \subseteq \mathrm{S} X$ is closed and invariant under $\gamma_{t}$ we call $F$ a subflow.

For any sliding block code $f: Y \rightarrow X$ we define $\mathrm{S} f: \mathrm{S} Y \rightarrow \mathrm{S} X$ in the obvious way, $[(y, t)] \mapsto[(f(y), t)]$, and say that $f$ is flow equivalent to $f^{\prime}: Y^{\prime} \rightarrow X^{\prime}$ when there are flow equivalences $\varphi, \psi$ with

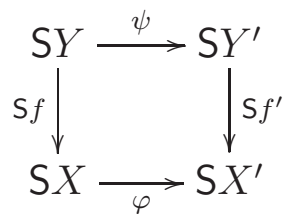

In this case, suppressing the domains and codomains from the notation, we write $[[f]]_{F E}=\left[\left[f^{\prime}\right]\right]_{F E}$.

Of course, any conjugacy $\varphi: Y \rightarrow X$ induces a flow equivalence $\mathrm{S} \varphi: \mathbf{S} Y \rightarrow \mathbf{S} X$, by the rule $\gamma_{t}(y) \mapsto \gamma_{t}(\varphi(y))$, for $y \in Y$ and $t \in \mathbb{R}$.

We describe next the other basic move we use for flow equivalence: symbol expansion.

Definition 2.3. Let $\mathfrak{a}$ be a finite alphabet and let $A \subseteq \mathfrak{a}$. Choose for each $a \in A$ a symbol $\star_{a}$ which does not belong to $\mathfrak{a}$. Let $\tilde{\mathfrak{a}}:=$ $\mathfrak{a} \cup\left\{\star_{a}: a \in A\right\}$. For $a \in \mathfrak{a}$ let

$$
\tilde{a}:= \begin{cases}a & \text { if } a \notin A, \\ a \star_{a} & \text { if } a \in A,\end{cases}
$$

and let $\iota_{A}$ be the map from $\mathfrak{a}^{\mathbb{Z}}$ to $\tilde{\mathfrak{a}}^{\mathbb{Z}}$ which maps

$$
\ldots a_{-2} a_{-1} . a_{0} a_{1} a_{2} \ldots
$$

to

$$
\ldots \tilde{a}_{-2} \tilde{a}_{-1} . \tilde{a}_{0} \tilde{a}_{1} \tilde{a}_{2} \ldots
$$


We will also use $\iota_{A}$ to denote the map from $\mathfrak{a}^{*}$ to $\tilde{\mathfrak{a}}^{*}$ which maps a word $a_{1} a_{2} \ldots a_{n}$ to $\tilde{a}_{1} \tilde{a}_{2} \ldots \tilde{a}_{n}$.

For a subshift $X$ of $\mathfrak{a}^{\mathbb{Z}}$ we will by $\mathfrak{e}_{A}(X)$ denote the shift space $\left\{\iota_{A}(x): x \in X\right\} \cup\left\{\sigma_{\tilde{\mathfrak{a}}}\left(\iota_{A}(x)\right): x \in X\right\}$.

Lemma 2.4. Let $X$ and $Y$ be shift spaces, let $\pi: Y \rightarrow X$ be a oneblock factor map and let $\mathcal{L}: \mathfrak{a}(Y) \rightarrow \mathfrak{a}(X)$ be the map that induces $\pi$. Let $A \subseteq \mathfrak{a}(X)$ and let $\tilde{A}:=\{b \in \mathfrak{a}(Y): \mathcal{L}(b) \in A\}$. Choose for each $a \in A$ a symbol $\star_{a}$ which does not belong to $\mathfrak{a}(X)$, and choose for each $b \in \tilde{A}$ a symbol $\star_{b}$ which does not belong to $\mathfrak{a}(Y)$. Let $\tilde{\mathcal{L}}$ be the map from $\mathfrak{a}(Y) \cup\left\{\star_{b}: b \in \tilde{A}\right\}$ to $\mathfrak{a}(X) \cup\left\{\star_{a}: a \in A\right\}$ that maps $c$ to $\mathcal{L}(c)$ for $c \in \mathfrak{a}(Y)$ and $\star_{b}$ to $\star_{\mathcal{L}(b)}$ for $b \in \tilde{A}$, and let $\mathfrak{e}_{A}(\pi)$ be the restriction of the one-block factor map induced by $\tilde{\mathcal{L}}$ to $\mathfrak{e}_{\tilde{A}}(Y)$.

Then $\mathfrak{e}_{A}(\pi)$ is a factor map from $\mathfrak{e}_{\tilde{A}}(Y)$ to $\mathfrak{e}_{A}(X)$, and the two factor maps $\pi$ and $\mathfrak{e}_{A}(\pi)$ are flow equivalent.

Proof. It is straightforward to check that $\mathfrak{e}_{A}(\pi)\left(\mathfrak{e}_{\tilde{A}}(Y)\right)=\mathfrak{e}_{A}(X)$.

Let $\varphi$ be the map from $\mathrm{S} X$ to $\operatorname{Se}_{A}(X)$ defined by

$\varphi([x, t])= \begin{cases}{\left[\iota_{A}(x), 2 t\right]} & \text { if } x_{0} \in A \text { and } t \in[0,1 / 2[, \\ {\left[\sigma_{\mathfrak{a}(T) \cup\left\{\star_{a}: a \in A\right\}}\left(\iota_{A}(x)\right), 2 t-1\right]} & \text { if } x_{0} \in A \text { and } t \in[1 / 2,1[, \\ {\left[\iota_{A}(x), t\right]} & \text { if } x_{0} \notin A,\end{cases}$

for $x \in X$ and $t \in\left[0,1\left[\right.\right.$, and let $\tilde{\varphi}$ be the map from $\mathrm{S} Y$ to $\operatorname{Se}_{\tilde{A}}(Y)$ defined by

$$
\tilde{\varphi}([y, t])= \begin{cases}{\left[\iota_{\tilde{A}}(y), 2 t\right]} & \text { if } y_{0} \in \tilde{A} \text { and } t \in[0,1 / 2[, \\ {\left[\sigma_{\mathfrak{a}(S) \cup\left\{\star_{b}: b \in \tilde{A}\right\}}\left(\iota_{\tilde{A}}(y)\right), 2 t-1\right]} & \text { if } y_{0} \in \tilde{A} \text { and } t \in[1 / 2,1[, \\ {\left[\iota_{\tilde{A}}(y), t\right]} & \text { if } y_{0} \notin \tilde{A},\end{cases}
$$

for $y \in Y$ and $t \in[0,1$. It is not difficult to check that $\varphi$ and $\tilde{\varphi}$ are flow equivalences and that the diagram

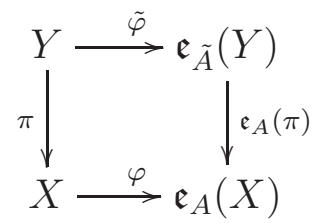

commutes. Thus $\pi$ and $\mathfrak{e}_{A}(\pi)$ are flow equivalent factor maps.

If $a \in \mathfrak{a}$, then we will write $\mathfrak{e}_{a}(\pi)$ instead of $\mathfrak{e}_{\{a\}}(\pi)$, and $\iota_{a}$ instead of $\iota_{\{a\}}$.

We will need to make frequent reference to the classical invariants of Parry and Sullivan [37] and Bowen and Franks [5], which were shown to be complete invariants of flow equivalence for infinite irreducible SFTs by Franks [20]. When a shift of finite type is given by an $n \times n$ adjacency matrix $A$, the invariant consists of the Bowen-Franks group $\operatorname{cok}(I-A)$ along with the sign of $\operatorname{det}(I-A)$. A zero entropy irreducible 
SFT is a cyclic permutation of finitely many points, whose mapping torus is a circle; in this case the Bowen-Franks group is $\mathbb{Z}$, which is also the Bowen-Franks group of some infinite SFTs.

\section{Multiplicity SETS OF CANONICAL COVERS}

We assume some familiarity with basic symbolic dynamics; 31 is an excellent basic reference. In this section we sketch some of the background.

Definition 3.1. By a cover we mean a pair of maps $\left(\mathfrak{c}, \pi_{\mathfrak{c}}\right)$ defined on the class of irreducible sofic shifts which to an irreducible sofic shift $X$ associate an irreducible shift of finite type $\mathfrak{c}(X)$ and a factor map $\pi_{\mathfrak{c}(X)}: \mathfrak{c}(X) \rightarrow X$

We will say that such a cover $\left(\mathfrak{c}, \pi_{\mathfrak{c}}\right)$ is canonical if the following holds: If $X_{1}$ and $X_{2}$ are irreducible sofic shifts and $\varphi: X_{1} \rightarrow X_{2}$ is a conjugacy, then there exists a unique conjugacy $\mathfrak{c}(\varphi): \mathfrak{c}\left(X_{1}\right) \rightarrow \mathfrak{c}\left(X_{2}\right)$ such that the diagram

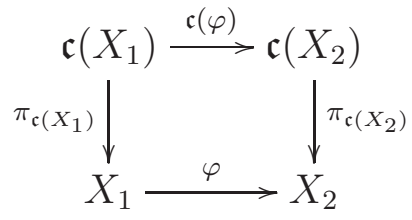

commutes.

We will say that a cover $\left(\mathfrak{c}, \pi_{\mathfrak{c}}\right)$ respects symbol expansion if the following holds: The factor map $\pi_{\mathfrak{c}(X)}$ is a one-block code for all irreducible sofic shifts $X$, and if $X$ is an irreducible sofic shift, $a \in$ $\mathfrak{a}(X), \mathcal{L}_{\mathfrak{c}(X)}: \mathfrak{a}(\mathfrak{c}(X)) \rightarrow \mathfrak{a}(X)$ is the map that induces $\pi_{\mathfrak{c}(X)}$, and $A=\left\{b \in \mathfrak{a}(\mathfrak{c}(X)): \mathcal{L}_{\mathfrak{c}(X)}(b)=a\right\}$, then there exists a conjugacy $\varphi: \mathfrak{c}\left(\mathfrak{e}_{a}(X)\right) \rightarrow \mathfrak{e}_{A}(\mathfrak{c}(X))$ such that the diagram

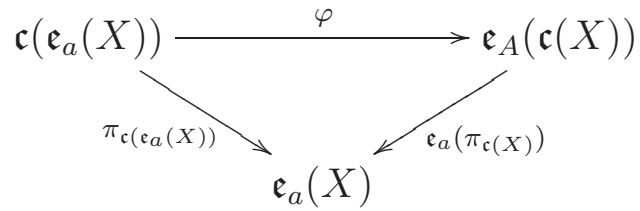

commutes

Krieger has in 29] proved that the right Fischer cover is canonical. We will now record that it also respects symbol expansion.

Proposition 3.2. The right Fischer cover respects symbol expansion.

Proof. Let $X$ be an irreducible sofic shift. The right Fischer cover of $X$ can be constructed as follows. Say that a word $u \in \mathrm{L}(X)$ (the language of $X$ ) is magic (also known as intrinsically synchronizing) if it has the following property: If $v u, u w \in \mathrm{L}(X)$, then $v u w \in \mathrm{L}(X)$. For a magic word $u$ let $\mathrm{F}(u):=\{v \in \mathrm{L}(X): u v \in \mathrm{L}(X)\}$, and let $(G, \mathcal{L})$ be the labeled graph with vertex set $\{\mathrm{F}(u): u \in \mathrm{L}(X)$ is magic $\}$ and where 
there for two magic words $u$ and $v$ and a symbol $a \in \mathfrak{a}(X)$ is an edge from $\mathrm{F}(u)$ to $\mathrm{F}(v)$ labeled $a$ if and only if $\mathrm{F}(v)=\mathrm{F}(u a)$. Then the right Fischer cover $(Y, \pi)$ of $X$ is the edge shift $Y$ of $G$ together with the one-block code $\pi$ induced by $\mathcal{L}$.

Let $a \in \mathfrak{a}(X)$. It is not difficult to check that we have

$$
\begin{aligned}
& \left\{u \in \mathrm{L}\left(\mathfrak{e}_{a}(X)\right): u \text { is magic }\right\} \\
& \quad=\left\{\star_{a} \iota_{a}(u), \iota_{a}(u) a, \iota_{a}(u) \in \mathrm{L}\left(\mathfrak{e}_{a}(X)\right): u \in \mathrm{L}(X) \text { is magic }\right\},
\end{aligned}
$$

and that if $u \in \mathrm{L}(X)$ is magic, then

$$
\mathrm{F}\left(\iota_{a}(u)\right)=\left\{\iota_{a}(v), \iota_{a}(w) a \in \mathrm{L}\left(\mathfrak{e}_{a}(X)\right): v, w \in \mathrm{F}(u)\right\},
$$

if $u \in \mathrm{L}(X)$ is magic and $\iota_{a}(u) a \in \mathrm{L}\left(\mathfrak{e}_{a}(X)\right)$, then

$$
\mathrm{F}\left(\iota_{a}(u) a\right)=\left\{\star_{a} \iota_{a}(v), \star_{a} \iota_{a}(w) a \in \mathrm{L}\left(\mathfrak{e}_{a}(X)\right): v, w \in \mathrm{F}(u a)\right\},
$$

and if $u \in \mathrm{L}(X)$ is magic and $\star_{a} \iota_{a}(u) \in \mathrm{L}\left(\mathfrak{e}_{a}(X)\right)$, then $\mathrm{F}\left(\star_{a} \iota_{a}(u)\right)=$ $\mathrm{F}\left(\iota_{a}(a u)\right)$. Thus if $(\hat{Y}, \hat{\pi})$ is the right Fischer cover of $\mathfrak{e}_{a}(X)$ and $\hat{\mathcal{L}}$ is the labeling which induces $\hat{\pi}$, and $A=\{e \in \mathfrak{a}(Y): \mathcal{L}(e)=a\}$, then there exists a bijection $\eta$ from $\mathfrak{a}\left(\mathfrak{e}_{A}(Y)\right)$ to $\mathfrak{a}(\hat{Y})$ which maps $e$ to the edge from $\mathrm{F}\left(\iota_{a}(u)\right)$ to $\mathrm{F}\left(\iota_{a}(v)\right)$ labeled $b$ if $e$ is the edge from $\mathrm{F}(u)$ to $\mathrm{F}(v)$ labeled $b$ and $b \neq a$, maps $e$ to the edge from $\mathrm{F}\left(\iota_{a}(u)\right)$ to $\mathrm{F}\left(\iota_{a}(u) a\right)$ labeled $a$ if $e$ is the edge from $\mathrm{F}(u)$ to $\mathrm{F}(u a)$ labeled $a$, and maps $\star_{e}$ to the edge from $\mathrm{F}\left(\iota_{a}(u) a\right)$ to $\mathrm{F}\left(\iota_{a}(u a)\right)$ labeled $\star_{a}$ if $e$ is the edge from $\mathrm{F}(u)$ to $\mathrm{F}(u a)$ labeled $a$. If $\tilde{\mathcal{L}}$ is the map from $\mathfrak{a}\left(\mathfrak{e}_{A}(Y)\right)$ to $\mathfrak{a}\left(\mathfrak{e}_{a}(X)\right)$ which maps $e$ to $\mathcal{L}(e)$ for $e \in \mathfrak{a}(Y)$ and $\star_{e}$ to $\star_{a}$ for $e \in A$, then $\hat{\mathcal{L}} \eta=\tilde{\mathcal{L}}$, and it follows that if $\varphi$ is the conjugacy from $\mathfrak{e}_{A}(Y)$ to $\hat{Y}$ induced by $\eta$, then the diagram

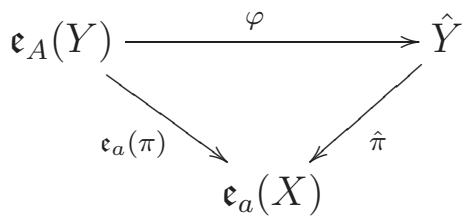

commutes.

One can in a similar way prove that the left Fischer cover, the right and the left Krieger cover, the predecessor set cover and the follower set cover all respect symbol expansion. It is shown in [29] that the left Fischer cover, the right and the left Krieger cover also are canonical.

We now have:

Theorem 3.3. Let $\left(\mathfrak{c}, \pi_{\mathfrak{c}}\right)$ be a cover which is canonical and respects symbol expansion. When $X_{1}$ and $X_{2}$ are flow equivalent irreducible sofic shifts the two factor maps $\pi_{\mathfrak{c}\left(X_{1}\right)}$ and $\pi_{\mathfrak{c}\left(X_{2}\right)}$ are flow equivalent factor maps. In other words,

$$
\left[\left[X_{1}\right]\right]_{F E}=\left[\left[X_{2}\right]\right]_{F E} \Longrightarrow\left[\left[\pi_{\mathfrak{c} X_{1}}\right]\right]_{F E}=\left[\left[\pi_{\mathfrak{c} X_{2}}\right]\right]_{F E} .
$$


Proof. Since flow equivalence among shift spaces is generated by conjugacy and symbol expansion, it is enough to prove that if $X_{1}$ and $X_{2}$ are conjugate, then $\pi_{\mathfrak{c}\left(X_{1}\right)}$ and $\pi_{\mathfrak{c}\left(X_{2}\right)}$ are conjugate factor maps, and that if $a \in \mathrm{L}\left(X_{1}\right)$ and $X_{2}=\mathfrak{e}_{a}\left(X_{1}\right)$, then $\pi_{\mathfrak{c}\left(X_{1}\right)}$ and $\pi_{\mathfrak{c}\left(X_{2}\right)}$ are flow equivalent factor maps. The first of these assertions is exactly the assertion that $\mathfrak{c}$ is canonical, and the second follows from the assumption that $\mathfrak{c}$ respects symbol expansion and Lemma 2.4.

Absolutely central to our approach in this paper will be the restriction of covers to their multiplicity sets.

Definition 3.4. Given a finite to one map $\pi: Y \rightarrow X$, we define

$$
\begin{aligned}
\text { MultiCard }(\pi) & =\left\{k \in \mathbb{N}: k>1 \text { and } \exists x \in X,\left|\pi^{-1}(x)\right|=k\right\}, \\
\mathrm{M}_{k}(\pi) & =\left\{x \in X:\left|\pi^{-1}(x)\right|=k\right\}, \\
\mathrm{M}_{k}^{-1}(\pi) & =\pi^{-1}\left(\mathrm{M}_{k}(\pi)\right), \\
\mathrm{M}(\pi) & =\bigcup_{k>1} \mathrm{M}_{k}(\pi), \\
\mathrm{M}^{-1}(\pi) & =\bigcup_{k>1} \mathrm{M}_{k}^{-1}(\pi) .
\end{aligned}
$$

We denote these sets as multiplicity sets and note that they are always shift invariant. Thus, they become a shift spaces in their own right precisely when they are closed. In this case, restricted factor maps such as

$$
\left.\pi\right|_{\mathrm{M}^{-1}}: \mathrm{M}^{-1}(\pi) \rightarrow \mathrm{M}(\pi)
$$

are defined. We note

Corollary 3.5. Let $\left(\mathfrak{c}, \pi_{\mathfrak{c}}\right)$ be a cover which is canonical, respects symbol expansion and is right or left resolving. When $X_{1}$ and $X_{2}$ are flow equivalent irreducible sofic shifts, then

$$
\operatorname{MultiCard}\left(\pi_{\mathfrak{c}\left(X_{1}\right)}\right)=\operatorname{MultiCard}\left(\pi_{\mathfrak{c}\left(X_{2}\right)}\right) .
$$

Proof. Because of Theorem 3.3 it suffices to prove that for such a cover $\pi: Y \rightarrow X$ we can infer whether $k \in \operatorname{MultiCard}(\pi)$ directly from the homeomorphism class of the map

$$
\mathrm{S} \pi: \mathrm{S} Y \rightarrow \mathrm{S} X \text {. }
$$

For this, we note that since the covers are either right or left resolving, we see that $y \in Y$ is periodic precisely when $\pi(y)$ is (although possibly with another period). Assume now that $k \in \operatorname{MultiCard}(\pi)$ and fix $\pi\left(y_{1}\right)=\cdots=\pi\left(y_{k}\right)=x$ with all $y_{i} \neq y_{j}, i \neq j$.

When $x$ is not periodic, neither is any of the $y_{i}$, and hence in $\mathrm{S} X$ there is a flow line homeomorphic to $\mathbb{R}$ having precisely $k$ distinct flow lines homeomorphic to $\mathbb{R}$ in its preimage. When $x$ is periodic, so are all 
$y_{i}$, and hence in $\mathrm{S} Y$ there are $m \leq k$ distinct flow lines homeomorphic to $\mathbb{T}$ mapping to the same flow line homeomorphic to $\mathbb{T}$. The winding numbers for each of these maps will be positive and sum up to $k$.

In the other direction, whenever one of these configurations can be found, $x$ and $y_{1}, \ldots, y_{k}$ can be constructed.

Remark 3.6. Note that for a general Fischer cover $\pi: Y \rightarrow X$ (even with $X$ AFT), it can happen that neither $\mathrm{M}^{-1}(\pi)$ nor $\mathrm{M}(\pi)$ is SFT ([13, pp. 60-61]).

\section{The extension theorem}

The proof of our extension result for flow equivalences of subflows is based on the following extension result for conjugacies of subshifts, which is a direct consequence of [14, Theorem 1.5].

Theorem 4.1. 14] Let $X$ be a mixing SFT with disjoint subshifts $Y$ and $Y^{\prime}$ such that

(1) There is a conjugacy $\varphi: Y \rightarrow Y^{\prime}$.

(2) For every positive integer $n, X \backslash Y$ contains at least two orbits of cardinality $n$.

Then there is a self-conjugacy $\widetilde{\varphi}: X \rightarrow X$ such that $\left.\widetilde{\varphi}\right|_{Y}=\varphi$. Moreover, $\widetilde{\varphi}$ can be chosen to act trivially on the dimension group of $X$.

We expect the "Moreover" statement of the following theorem (Theorem 4.2), regarding the induced isomorphism on the isotopy futures group, $\mathcal{F}(X)$, may be useful for future coding arguments, but we do not use it for the flow equivalence results in the current paper. So we postpone a short review of $\mathcal{F}(X)$ to the end of this section.

Theorem 4.2. (Extension Theorem) Suppose $X$ and $X^{\prime}$ are flow equivalent irreducible SFTs with proper subsystems $Y, Y^{\prime}$ which are flow equivalent through

$$
\varphi: \mathrm{S} Y \rightarrow \mathrm{S}^{\prime}
$$

Then there is a flow equivalence

$$
\widetilde{\varphi}: \mathrm{S} X \rightarrow \mathrm{S} X^{\prime}
$$

which agrees with $\varphi$ on $\mathrm{SY}$.

Moreover, given an isomorphism $b: \mathcal{F}(X) \rightarrow \mathcal{F}\left(X^{\prime}\right), \widetilde{\varphi}$ can be chosen such that the isomorphism $\mathcal{F}(X) \rightarrow \mathcal{F}\left(X^{\prime}\right)$ induced by $\widetilde{\varphi}$ equals $b$.

The condition of properness is necessary, as a proper subshift $X^{\prime} \subset X$ may be flow equivalent to $X$ without the embedding extending.

Proof of Theorem 4.2. We will prove the theorem in several steps. Every step but the last is a reduction to a special case. We give a complete proof of the existence of $\widetilde{\varphi}$ before discussing the action on the isotopy futures group. We will use Lemma 4.3 which we state and prove just after this proof. 
Step 1: Reduction to the case $X=X^{\prime}$.

Let $\psi: \mathrm{S} X \rightarrow \mathbf{S} X^{\prime}$ be a flow equivalence. Suppose there is a flow equivalence $\kappa: \mathrm{S} X \rightarrow \mathrm{S} X$ which is an extension of the flow equivalence $\psi^{-1} \varphi: \mathrm{S} Y \rightarrow \mathrm{S} Y$. Then $\psi \kappa: \mathrm{S} X \rightarrow \mathrm{S} X^{\prime}$ is a flow equivalence extending $\varphi: \mathrm{S} Y \rightarrow \mathrm{S}^{\prime}$.

So from here, without loss of generality we assume $X=X^{\prime}$. Also, set $Y_{1}=Y$ and $Y_{2}=Y^{\prime}$.

Step 2: Reduction to the case $X$ is mixing.

There is a flow equivalence $\gamma: \mathbf{S} X \rightarrow \mathbf{S} X^{\prime \prime}$ where $X^{\prime \prime}$ is a mixing SFT (this is most easily seen by appealing to [20]). As in Step 1, if we can find a flow equivalence $\mathrm{S} X^{\prime \prime} \rightarrow \mathrm{S} X^{\prime \prime}$ extending $\gamma \varphi \gamma^{-1}: \mathrm{S}\left(\gamma Y_{1}\right) \rightarrow$ $\mathrm{S}\left(\gamma Y_{2}\right)$, then we can pull it back to a flow equivalence $\mathrm{S} X \rightarrow \mathrm{S} X$ extending $\varphi$.

Step 3: Reduction to the case $X$ contains a subshift $Y_{3}$ conjugate to the 3-shift, and the subshifts $Y_{1}, Y_{2}, Y_{3}$ of $X$ are pairwise disjoint.

Given $N$ and $W=Y_{1} \cup Y_{2}$ in $X$, we take $\gamma, g$ and $B$ as given by Lemma 4.3. We choose $N$ large enough that a disjoint union of the subshift $Y_{2}$ and two copies of the 3 -shift embed in $X_{N}$ (the full $N$ shift). The subshift $g\left(Y_{2}\right)$ is conjugate to $Y_{2}$, so there is an embedding $f$ of $g\left(Y_{2}\right)$ into $X_{B(22)}=X_{N}$ with image disjoint from a copy $Y_{3}$ of the 3-shift contained in $X_{N}$.

By Theorem 4.1, there is an automorphism $k$ of $X_{B}$ which restricts to $f$ on $g\left(Y_{2}\right)$ and restricts to the identity on $Y_{3}$. (The extra copy of the 3-shift provides enough periodic orbits.) Let $\kappa=\mathrm{S} k: \mathrm{S} X_{B} \rightarrow \mathrm{S} X_{B}$. Now $\mathrm{S}\left(g\left(Y_{1}\right)\right), \mathbf{S}\left(\kappa g\left(Y_{2}\right)\right)$ and $\mathbf{S} Y_{3}$ are pairwise disjoint in $\mathbf{S} X_{B}$.

Suppose we can find a flow equivalence $\alpha: \mathrm{S} X_{B} \rightarrow \mathrm{S} X_{B}$ which extends the flow equivalence $\kappa \gamma \varphi \gamma^{-1}: \mathrm{S}\left(g\left(Y_{1}\right)\right) \rightarrow \mathrm{S}\left(\kappa g\left(Y_{2}\right)\right)$. Then $\gamma^{-1} \kappa^{-1} \alpha \gamma$ is a flow equivalence $\mathrm{S} X \rightarrow \mathrm{S} X$ which extends $\varphi$. So, without loss of generality we may assume that $X$ contains a subshift $Y_{3}$ conjugate to the 3-shift, with $Y_{1}, Y_{2}$ and $Y_{3}$ pairwise disjoint.

Step 4: Reduction to the case $\varphi=\mathrm{Sh}: Y_{1} \rightarrow Y_{2}$, with $h: Y_{1} \rightarrow Y_{2}$ a topological conjugacy.

By the Parry-Sullivan argument in [37] (see [8, Theorem 4.2] for full details), there are closed sets $D_{1} \subseteq Y_{1}$ and $D_{2} \subseteq Y_{2}$; a homeomorphism $h: D_{1} \rightarrow D_{2}$; and a flow equivalence $\epsilon: \mathrm{S} Y_{1} \rightarrow \mathrm{S} Y_{1}$ isotopic to the identity such that the following hold:

(1) For $k=1,2: D_{k}$ is a cross section of $\mathrm{S} Y_{k}$

(2) The restriction $\left.(\varphi \epsilon)\right|_{D_{1}}$ is a topological conjugacy of the return maps $\rho_{i}: D_{i} \rightarrow D_{i}$ (i.e. $\varphi \epsilon\left(D_{1}\right)=D_{2}$ and $\left.(\varphi \epsilon)\right|_{D_{1}} \rho_{1}=$ $\left.\left.\rho_{2}(\varphi \epsilon)\right|_{D_{1}}\right)$.

Let $k$ be 1 or 2 . Then $D_{k}$ is a cross section for $\mathrm{S} Y_{k}$ and $D_{k} \subseteq Y_{k}$. Therefore, $D_{k}$ is clopen in $Y_{k}$ and $Y_{k}$ is a discrete tower over $D_{k}$. For $x \in D_{k}$, define $\tau_{k}(x)=\min \left\{j>0: \sigma^{j}(x) \in D_{k}\right\}$, the first return time 
to $D_{k}$. Set $T_{k}=\max \tau_{k}$ and $D_{k}(j)=\left\{x \in D_{k}: \tau_{k}(x)=j\right\}$. Then

$$
\left\{\sigma^{i}\left(D_{k}(j)\right): 1 \leq j \leq T_{k}, 0 \leq i<j\right\}
$$

is a clopen partition of $Y_{k}$ (some of the sets $D_{k}(j)$ might be empty). For $1 \leq j \leq T_{k}$, choose $\widetilde{D}_{k}(j)$ a clopen subset of $X$ such that $D_{k}(j) \subseteq \widetilde{D}_{k}(j)$ and $\left\{\sigma^{i}\left(\widetilde{D}_{k}(j)\right): 1 \leq j \leq T_{k}, 0 \leq i<j\right\}$ is a collection of pairwise disjoint sets. Set $\widetilde{D}_{k}=\bigcup_{j=1}^{T_{k}} \widetilde{D}_{k}(j)$ and $\widetilde{E}_{k}=\bigcup_{j=1}^{T_{k}} \bigcup_{i=1}^{j-1} \sigma^{i}\left(\widetilde{D}_{k}(j)\right)$. Because the subshifts $Y_{1}, Y_{2}, Y_{3}$ are pairwise disjoint, we may also require

$$
\left(\widetilde{D}_{1} \cup \widetilde{E}_{1}\right) \cap\left(\widetilde{D}_{2} \cup \widetilde{E}_{2}\right)=\emptyset,
$$

and

Define

$$
\left(\bigcup_{k=1}^{2}\left(\widetilde{D}_{k} \cup \widetilde{E}_{k}\right)\right) \cap Y_{3}=\emptyset
$$

$$
\widetilde{D}_{k}=X \backslash \bigcup_{k=1}^{2}\left(\widetilde{D}_{k} \cup \widetilde{E}_{k}\right)
$$

and

$$
\widetilde{D}=\bigcup_{k=1}^{3} \widetilde{D}_{k}
$$

Now $\widetilde{D}$ is a discrete cross section for $X$ (and a cross section for $\mathrm{S} X$ ). Let $\widetilde{\rho}: \widetilde{D} \rightarrow \widetilde{D}$ be the return map under the shift $\sigma$ (or equivalently, under the suspension flow on $\mathrm{S} X)$. If, for $k \in\{1,2\}, x \in D_{k}$, then $\widetilde{\rho}(x)=\rho_{k}(x)$, and if $x \in Y_{3}$, then $\widetilde{\rho}(x)=\sigma(x)$.

Because $\widetilde{D}$ is a discrete cross section of the SFT $X$, there is a shift of finite type $X^{\prime \prime}$ and a flow equivalence $\mathbf{S} X \rightarrow \mathbf{S} X^{\prime \prime}$ such that $\left.\gamma\right|_{\widetilde{D}}$ is a topological conjugacy from $(\widetilde{D}, \widetilde{\rho})$ to $X^{\prime \prime}$. For $k \in\{1,2\}$, set $Y_{k}^{\prime \prime}=$ $\gamma\left(D_{k}\right)$, and set $Y_{3}^{\prime \prime}=\gamma\left(Y_{3}\right)$. Because $Y_{3} \subseteq \widetilde{D}$, the restriction $\left.\gamma\right|_{Y_{3}}: Y_{3} \rightarrow$ $Y_{3}^{\prime \prime}$ is a topological conjugacy of subshifts, and $Y_{3}^{\prime \prime}$ is conjugate to the 3-shift.

Let $\varphi^{\prime \prime}=\gamma \varphi \epsilon \gamma^{-1}: \mathrm{S} Y_{1}^{\prime \prime} \rightarrow \mathrm{S} Y_{2}^{\prime \prime}$ and $h^{\prime \prime}=\left.\varphi^{\prime \prime}\right|_{Y_{1}^{\prime \prime}}$. Because $\left.\gamma\right|_{D_{k}}$ conjugates $\left(D_{k}, \rho_{k}\right)$ and $Y_{k}^{\prime \prime}$, and $\varphi \epsilon$ conjugates $\left(D_{1}, \rho_{1}\right)$ and $\left(D_{2}, \rho_{2}\right)$, it follows that $h^{\prime \prime}$ defines a conjugacy of shifts $Y_{1}^{\prime \prime} \rightarrow Y_{2}^{\prime \prime}$. There is therefore a flow equivalence $\epsilon^{\prime \prime}: \mathrm{S} Y_{1}^{\prime \prime} \rightarrow \mathrm{S} Y_{1}^{\prime \prime}$ isotopic to the identity such that $\varphi^{\prime \prime} \epsilon^{\prime \prime}=S h^{\prime \prime}$. If we can find $\kappa: S X^{\prime \prime} \rightarrow \mathrm{S} X^{\prime \prime}$ a flow equivalence extending $\varphi^{\prime \prime} \epsilon^{\prime \prime}=\mathrm{S} h^{\prime \prime}$, then we have that $\gamma^{-1} \kappa \gamma: \mathrm{S} X \rightarrow \mathrm{S} X$ is a flow equivalence extending $\varphi \epsilon \gamma^{-1} \epsilon^{\prime \prime} \gamma$. Since $\epsilon \gamma^{-1} \epsilon^{\prime \prime} \gamma: \mathrm{S} Y_{1} \rightarrow \mathrm{S} Y_{1}$ is a flow equivalence which is isotopic to the identity, it follows from [8, Proposition 7.1] that there is a flow equivalence $\widetilde{\epsilon}: \mathrm{S} X \rightarrow \mathrm{S} X$ which is isotopic to the identity such that $\tilde{\epsilon}$ equals $\epsilon \gamma^{-1} \epsilon^{\prime \prime} \gamma$ on $S Y_{1}$. Thus, $\gamma^{-1} \kappa \gamma \widetilde{\epsilon}^{-1}: \mathrm{S} X \rightarrow \mathrm{S} X$ is a flow equivalence extending $\varphi$.

So without loss of generality, in the next step we may assume there is a topological conjugacy of subshifts $h: Y_{1} \rightarrow Y_{2}$ such that $\varphi=$ Sh: $Y_{1} \rightarrow Y_{2}$. 
Step 5: Appeal to Extension Theorem for conjugacy.

We have $\varphi=\mathrm{S} h: \mathrm{S} Y_{1} \rightarrow \mathrm{S} Y_{2}$ with $h: Y_{1} \rightarrow Y_{2}$ a conjugacy of subshifts of the mixing SFT $X$. For every $k$ in $\mathbb{N}$ the set $X \backslash Y_{1}$ contains at least two $X$-orbits of cardinality $k$ (because this set contains $Y_{3}$, a copy of the 3 -shift). It follows from Theorem 4.1 that $h$ extends to a conjugacy $k: X \rightarrow X$. Then the flow equivalence $\mathrm{S} k: \mathrm{S} X \rightarrow \mathrm{S} X$ is an extension of $\varphi=\mathrm{S} h: Y_{1} \rightarrow Y_{2}$. This finishes the proof that the flow equivalence extending $\varphi$ exists.

Now we turn to the "Moreover" claim. We assume the background given at the end of this section. For a flow equivalence $\beta$, we use $[\beta]$ to denote the induced isomorphism of isotopy futures groups (an automorphism if $\beta$ is a self equivalence). We claim that the extension $\widetilde{\varphi}$ produced in Steps 2-5 acts trivially on $\mathcal{F}(X)$, for the following reasons.

(1) The automorphisms $k$ of Steps 3 and 5 , provided by Theorem 4.1. are chosen to be inert, so $[\mathrm{S} k]=\mathrm{Id}$.

(2) If $[\beta]=\mathrm{Id}$, then $\left[\gamma \beta \gamma^{-1}\right]=[\gamma][\beta]\left[\gamma^{-1}\right]=\mathrm{Id}$.

(3) For a flow equivalence $\widetilde{\epsilon}$ isotopic to the identity, $[\widetilde{\epsilon}]=\mathrm{Id}$.

Now suppose $b: \mathcal{F}(X) \rightarrow \mathcal{F}\left(X^{\prime}\right)$ is an isomorphism of isotopy futures groups and we want the flow equivalence $\mathrm{S} X \rightarrow \mathrm{S} X^{\prime}$ extending $\varphi: \mathrm{S} Y \rightarrow \mathrm{S} Y^{\prime}$ to induce the isomorphism $b$. Let $\psi: \mathrm{S} X \rightarrow \mathrm{S} X^{\prime}$ be a flow equivalence. Let $a: \mathcal{F}(X) \rightarrow \mathcal{F}(X)$ be an automorphism such that $b=[\psi] a$. By [7, Theorem 7.13], there is a flow equivalence $\alpha: S_{X} \rightarrow S_{X}$ such that $[\alpha]=a$. Now $\alpha^{-1} \psi^{-1} \varphi$ defines a flow equivalence from $S Y$ to a submapping torus of SX. Apply the argument of Steps 2-5 to extend this to a flow equivalence $\gamma: \mathbf{S} X \rightarrow \mathbf{S} X$ such that $[\gamma]=\mathrm{Id}$. Define $\widetilde{\varphi}=\psi \alpha \gamma$. Then $\bar{\varphi}$ is a flow equivalence $\mathrm{S} X \rightarrow \mathrm{S} X^{\prime}$ extending $\varphi$ such that $[\widetilde{\varphi}]=[\psi][\alpha]=[\psi] a=b$. This completes the proof.

Now comes the lemma that we use in the proof of Theorem 4.2 .

Lemma 4.3. Suppose $X$ is a mixing SFT and $W$ is a proper subshift of $X$ and $N$ is a positive integer. Then there is a primitive matrix $B$ with $2 \times 2$ block form $B=\left(\begin{array}{cc}B(11) & B(12) \\ B(21) & N\end{array}\right)$ and a flow equivalence $\gamma: \mathrm{S} X \rightarrow \mathrm{S} X_{B}$ and a topological conjugacy $g$ from $W$ to a subshift of $X_{B(11)}$ such that the restriction of $\gamma$ to $\mathrm{S} W$ equals $\mathrm{S} g$.

Proof. Given integers $k \geq 2$ and $N$, let $Q_{k, N}$ be the $k \times k$ matrix $Q$ such that $Q(i, i+1)=1,1 \leq i<k ; Q(k, 1)=N$; and $Q(i, j)=0$ otherwise.

Let $W^{\prime} \neq X$ be a mixing SFT such that $W \subseteq W^{\prime} \subset X$. Let $C$ be a primitive matrix such that $X_{C}$ is topologically conjugate to $W^{\prime}$. Given $N$, fix $k$ such that with $Q=Q_{k, N}$ and $E=\left(\begin{array}{ll}C & 0 \\ 0 & Q\end{array}\right)$, it follows from Krieger's Embedding Theorem that there is an embedding $\eta: X_{E} \rightarrow X$ with $\eta\left(X_{E}\right) \neq X$. Using a modification of Krieger's Embedding Theorem proof [6, Remark p.548], we require $\eta$ to be an extension of the given conjugacy from $X_{C}$ to $W^{\prime}$. 
Next we let $\gamma$ be a conjugacy from $X$ to a higher block presentation $X_{D}$ of $X$, where $D$ is a primitive matrix with a principal submatrix $D(1)$ such that $\gamma$ maps $\eta\left(X_{E}\right)$ onto $X_{D(1)}$.

Next we will appeal to Nasu's Masking Lemma which can be stated and proved in terms of graphs (as in [35, Lemma 3.18] and [31, Sec. 10.2]) or matrices (as in [10, Appendix 1]). The matrix statement gives that if $M$ is a principal submatrix of a square matrix $A$ over $\mathbb{Z}_{+}$, and a strong shift equivalence over $\mathbb{Z}_{+}$from $M$ to a matrix $M^{\prime}$ is given, then it can be extended to a strong shift equivalence over $\mathbb{Z}_{+}$from $A$ to some matrix in which $M^{\prime}$ is a principal submatrix. As a consequence in our case, there is a primitive matrix $A$ with block form

$$
A=\left(\begin{array}{lll}
A(11) & A(12) & A(13) \\
A(21) & A(22) & A(23) \\
A(31) & A(32) & A(33)
\end{array}\right)
$$

with

$$
\left(\begin{array}{ll}
A(22) & A(23) \\
A(32) & A(33)
\end{array}\right)=\left(\begin{array}{cc}
C & 0 \\
0 & Q
\end{array}\right)=E
$$

such that there is a topological conjugacy $h: X \rightarrow X_{A}$ such that the following holds. Identify

$$
X\left(\begin{array}{ll}
A(22) & A(23) \\
A(32) & A(33)
\end{array}\right)
$$

and $X_{E}$; then the restriction of $h$ to $X_{D(1)}$ is $(\gamma \eta)^{-1}$. It follows that $h\left(W^{\prime}\right)=X_{A(22)} \subset X_{A}$, so $h$ maps $W$ into $X_{A(22)}$.

Let $A$ be $m \times m$. Set $A(1)=A$. In the order $j=1,2, \ldots, k-1$ define $I-A(j+1)$ to be the matrix obtained from $I-A(j)$ by adding column $m-k+j$ of $I-A(j)$ to column $m-k+j+1$. For each $j$, this is a positive matrix equivalence giving a flow equivalence $\varphi_{j}: \mathrm{S} X_{A(j)} \rightarrow \mathrm{S} X_{A(j+1)}$ which is the identity on the submapping torus $S X_{M}$, where

$$
M=\left(\begin{array}{ll}
A(11) & A(12) \\
A(21) & A(22)
\end{array}\right) .
$$

Set $B=A(j+k-1)$. Then $B(m, m)=N$ and the composition

$$
\varphi_{k-1} \cdots \varphi_{2} \varphi_{1}(\mathrm{~S} h)(\mathrm{S} \gamma): \mathrm{S} X \rightarrow \mathrm{S} X_{B}
$$

is the desired flow equivalence.

Remark 4.4. There is an alternate proof of Lemma 4.3 which constructs $B$ using a sequence of flow equivalence arguments from the proofs of [20, Lemma 2.1, Corollary 2.3, Theorem 2.4].

We turn now to a brief review of the isotopy futures group.

There is a homomorphism (the dimension representation) $\rho_{A}$ from the automorphism group of an $\mathrm{SFT} X_{A}$ to the group of automorphisms of its dimension group. An automorphism in the kernel of $\rho_{A}$ is called inert; it acts by the identity on the dimension group. We are using the 
dimension group built from left infinite rays; for background on this, see 15 .

The mapping class group of an SFT $X_{A}$ is the group of orientation preserving homeomorphisms of its mapping torus $\mathrm{S} X_{A}$, modulo isotopy. There is a group associated to $S X_{A}$ which is the flow equivalence analogue of the association of the dimension group to $X_{A}$ : the isotopy futures group, $\mathcal{F}\left(X_{A}\right)$. This group is the free abelian group with generators the set of rays $x(-\infty, n], x \in X_{A}$ and $n \in \mathbb{Z}$, given certain relations. The map which sends a ray $x(-\infty, n]$ to the vector $e_{j}$ such that $j$ is the terminal vertex of $x_{n}$ induces an isomorphism from $\mathcal{F}\left(X_{A}\right)$ to $\operatorname{cok}(I-A)$. The construction is very similar to Krieger's construction of the dimension group out of rays. There is also a flow analogue of the dimension representation: a flow equivalence $\mathrm{S} X_{A} \rightarrow \mathrm{S} X_{A}$, by its action on finite unions of rays, induces an automorphism of $\mathcal{F}\left(X_{A}\right)$. An inert automorphism $U$ of $X_{A}$ induces a flow equivalence of $\mathrm{S} X_{A}$ which acts by the identity on $\mathcal{F}\left(X_{A}\right)$, because the action of $\mathrm{S} U$ on $\mathcal{F}\left(X_{A}\right)$ factors through the action of $U$ the dimension group.

See [7, Section 7] for the development of isotopy futures theory, and [8] for more on isotopy and the mapping class group of a shift of finite type.

\section{THE REDUCTION THEOREM FOR AFT SHIFTS}

We are ready to state our main result which reduces the question of flow equivalence of AFT sofic shifts to a question of flow equivalence of certain covers.

Definition 5.1. The shift space $X$ is said to be almost finite type if there is an irreducible subshift of finite type $Y$ and a factor map $\pi: Y \rightarrow X$ that is one-to-one on a nonempty open set.

The AFT shifts, originally introduced by Marcus [32, Definition 4] to address practical coding problems, have emerged as a natural and large class of relatively tractable sofic shifts [31, Sec.13]. AFT shifts have a variety of characterizations, collected below in Theorem 5.2.

Theorem 5.2. Let $X$ be an irreducible, strictly sofic shift. The following are equivalent

(i) The shift $X$ is AFT.

(ii) The left Fischer cover of $X$ is right-closing.

(iii) The right Fischer cover of $X$ is left-closing.

(iv) $X$ has a minimal cover (i.e. an SFT $Y$ and a factor map $\pi: Y \rightarrow$ $X$ such that any other factor map $\varphi: Y^{\prime} \rightarrow X$ (from an SFT $Y^{\prime}$ onto X) must factor through $\pi$ ) [12, 41]. (This cover must be conjugate to the left and right Fischer covers.)

(v) The right and left Fischer covers of $X$ are topologically conjugate as factor maps. 
(vi) $X$ is the factor of an SFT by a biclosing map.

(vii) The multiplicity set of its (left or right) Fischer cover is a proper subshift of the domain.

We draw the reader's attention to (vii) in particular, since it will allow us to work with multiplicity sets such as $\mathrm{M}(\pi)$ and $\mathrm{M}^{-1}(\pi)$ as shift spaces in their own right. Because the left and right Fischer covers of an AFT shift are conjugate, we may (when concerned only with the conjugacy class of the Fischer cover) refer to the Fischer cover of an AFT shift.

Theorem 5.3. (Reduction Theorem) For $i=1,2$, let $\pi_{i}: Y_{i} \rightarrow X_{i}$ be the Fischer cover of an AFT shift $X_{i}$. Then the following are equivalent:

(1) $X_{1}$ and $X_{2}$ are flow equivalent.

(2) The two factor maps $\pi_{1}$ and $\pi_{2}$ are flow equivalent factor maps.

(3) $Y_{1}$ and $Y_{2}$ are flow equivalent and the restricted factor maps $\left.\left(\pi_{1}\right)\right|_{\mathrm{M}^{-1}}$ and $\left.\left(\pi_{2}\right)\right|_{\mathrm{M}^{-1}}$ are flow equivalent factor maps.

Proof. It is obvious that (2) implies (3). It is proven in 29] that the Fischer cover is canonical, and it follows from Proposition 3.2 that it also respects symbol expansion. The domain of each Fischer cover $\pi_{i}$ is an irreducible SFT, in which $\mathrm{M}^{-1}\left(\pi_{i}\right)$ is a proper subshift [13]. Thus all of the assumptions of Theorem 3.3 are satisfied, and (1) implies (2). It remains to prove that (3i) implies (11).

Suppose then that condition (3) holds. Then there are flow equivalences $\varphi, \psi$ giving a commuting central square in the following diagram

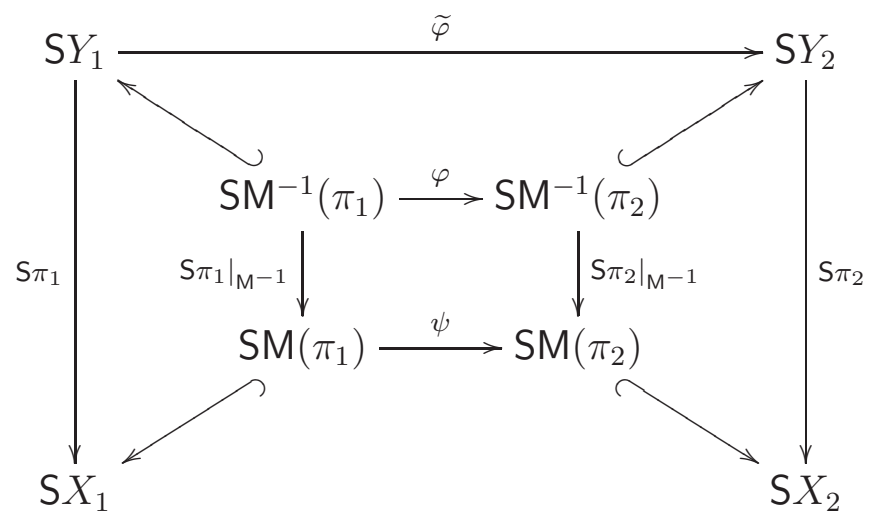

in which the hooked arrows are inclusions. By the Extension Theorem 4.2, there exists a flow equivalence $\widetilde{\varphi}: \mathrm{S} Y_{1} \rightarrow \mathrm{S} Y_{2}$ extending $\varphi$, so that the entire diagram commutes. Because the homeomorphism $\widetilde{\varphi}$ takes the quotient relation of $S \pi_{1}$ to that of $S \pi_{2}$, it induces a homeomorphism $\mathrm{S} X_{1} \rightarrow \mathrm{S} X_{2}$, which is easily seen to be a flow equivalence. Hence (3) implies (1). 
Next, as an immediate application of the Reduction Theorem 5.3 we classify the irreducible near Markov shifts up to flow equivalence.

Definition 5.4. [13] An irreducible sofic shift space $X$ is near Markov if one of its Fischer covers $\pi: Y \rightarrow X$ has a finite multiplicity set $\mathrm{M}^{-1}(\pi)$.

A near Markov shift is AFT, so as noted above, we can refer to the Fischer cover (up to conjugacy of factor maps).

Proposition 5.5. If $X$ is flow equivalent to $X^{\prime}$, and $X$ is irreducible near Markov, then so is $X^{\prime}$.

Proof. Because $X$ is an AFT shift, so is $X^{\prime}$ 21]. The irreducible near Markov shifts are precisely the AFT sofic shifts for which the mapping torus of the multiplicity set $\mathrm{M}^{-1}(\pi)$ of the Fischer cover $\pi$ is a finite union of circles, so the conclusion follows from Corollary 3.5 .

Definition 5.6. The multiplicity graph $G[\pi]$ of a factor map $\pi: Y \rightarrow X$ with $\mathrm{M}^{-1}(\pi)$ finite is a bipartite graph defined as follows. Organize the finite sets $\mathrm{M}^{-1}(\pi)$ and $\mathrm{M}(\pi)$ into orbits $o_{1}, \ldots o_{k}$ and $v_{1}, \ldots, v_{\ell}$ and let $j(i)$ be the $j$ such that $\pi\left(o_{i}\right)=v_{j}$. Note that in this case, $\left|v_{j(i)}\right|$ must divide $\left|o_{i}\right|$, and set

$$
w(i)=\frac{\left|o_{i}\right|}{\left|v_{j(i)}\right|} .
$$

The graph $G[\pi]$ then has vertices $\left\{y_{1}, \ldots, y_{k}, x_{1}, \ldots x_{\ell}\right\}$ with $w(i)$ edges from $y_{i}$ to $x_{i}$ for each $i \in\{1, \ldots k\}$.

Lemma 5.7. The multiplicity graph is a complete flow invariant for the class of maps $\left.\pi\right|_{\mathrm{M}^{-1}}(\pi)$ such that $\mathrm{M}^{-1}(\pi)$ is finite.

Proof. The mapping tori for finite shifts $\mathrm{M}^{-1}(\pi)$ and $\mathrm{M}(\pi)$ are finite unions of circles. A circle $\mathrm{S}_{i}$ in $\mathrm{SM}^{-1}(\pi)$ is wrapped by $\mathrm{S} \pi w(i)$ times around its image circle $\mathbf{S} v_{j(i)}$; this (winding number) $w(i)$ is a complete invariant of flow equivalence of the map $\left.\mathrm{S} \pi\right|_{S_{o_{i}}}$. Hence the multiplicity graph encodes an invariant of flow equivalence, which is easily checked to be complete, for this class. The conclusion follows by Theorem 5.3 .

Definition 5.8. For a near Markov shift $X$ with Fischer cover $\pi: Y \rightarrow$ $X$ where $Y=Y_{A}$ for a matrix $A$, we define $\mathfrak{I}(X)$ as the collection of data

$$
\left(\operatorname{BF}\left(Y_{A}\right), \operatorname{det}(I-A), G[\pi]\right) .
$$

Theorem 5.9. For a pair of near Markov shifts $X, X^{\prime}$ we have

$$
[[X]]_{F E}=\left[\left[X^{\prime}\right]\right]_{F E} \Longleftrightarrow \mathfrak{I}(X) \simeq \mathfrak{I}\left(X^{\prime}\right) .
$$


Proof. We may apply Theorem 5.3 since $\mathrm{M}^{-1}(\pi)$ is closed by Theorem 5.2 (vii). Now the first component of our invariant is a complete invariant of flow equivalence of the Fischer cover by Franks's classification ([20]), and the latter is a complete invariant of the multiplicity cover by Lemma 5.7 .

Franks proved in [20] that any irreducible SFT is flow equivalent to its time-reversal, by noting that his complete invariant did not distinguish them. This fails dramatically even for general AFT shifts; e.g., for the Fischer cover $\pi$, we could arrange $\mathrm{M}(\pi)$ to be the SFT presented by the matrix $\left(\begin{array}{ll}2 & 1 \\ 0 & 1\end{array}\right)$. However, Franks' result carries over to near Markov shifts.

Corollary 5.10. A near Markov shift is flow equivalent to its timereversal.

Proof. The complete invariant is the same for the system and its timereversal.

\section{N-POINT EXTENSIONS AND $G$-SFTs}

For a set $E$, let $\mathcal{S}_{E}$ be the group of permutations of $E$, with the group product $g h$ defined by $g h: x \mapsto g(h(x))$ (i.e., $h$ acts first). Let $\mathcal{S}_{N}$ denote $\mathcal{S}_{E}$ with $E=\{1, \ldots, N\}$.

In this section we recall how to reduce the classification up to topological conjugacy of $N$-point extensions of SFTs to the classification of related $G$-SFTs with $G=\mathcal{S}_{N}$. This reduction will be used for flow equivalence results in Section 7 . The reduction is due to Adler, Kitchens and Marcus [1, 2] (adapting ideas of Rudolph [40]).

Recall that factor maps $\pi: Y \rightarrow X$ and $\pi^{\prime}: Y^{\prime} \rightarrow X^{\prime}$ are defined to be isomorphic (topologically conjugate) if there are topological conjugacies $\alpha, \beta$ such that $\pi^{\prime} \alpha=\beta \pi$. Equivalently, there is a topological conjugacy $\alpha: Y \rightarrow Y^{\prime}$ such that for all $w, y$ in $Y: \pi(w)=\pi(y)$ if and only if $\pi^{\prime}(\alpha(w))=\pi^{\prime}(\alpha(y))$.

Definition 6.1. Suppose $N$ is a positive integer and $\sigma: X \rightarrow X$ is a homeomorphism of a compact metric space. Let $Y=X \times\{1, \ldots, N\}$ and $\rho: Y \rightarrow Y$ be a homeomorphism of the form

$$
\rho:(x, k) \mapsto\left(\sigma(x), \tau_{x}(k)\right),
$$

with $\tau_{x} \in \mathcal{S}_{N}$ acting from the left (e.g., $\left.\rho^{2}:(x, k) \mapsto\left(\sigma^{2}(x), \tau_{\sigma(x)} \tau_{x}(k)\right)\right)$. Then the factor map $(Y, \rho) \mapsto(X, \sigma)$ defined by $(x, k) \mapsto x$ is an $N$ point extension of $(X, \sigma)$. A factor map $(Y, \rho) \rightarrow(X, \sigma)$ is an $N$-point extension of $(X, \sigma)$ if and only if it is isomorphic to such a factor map. When $(Y, \rho)$ is the domain system of an $N$-point extension of $(X, \sigma)$, $(Y, \rho)$ itself is sometimes referred to as an $N$-point extension of $(X, \sigma)$. The function $\tau$ is called the skewing function.

Facts 6.2. We mention some routinely verified facts. 
(1) The map $x \mapsto \tau_{x}$ from Definition 6.1 is a continuous function $\tau: X \rightarrow \mathcal{S}_{N}$

(2) A continuous, constant $N$-to-1 factor map $\pi:(Y, \rho) \rightarrow(X, \sigma)$ is an $N$-point extension of $(X, \sigma)$ if and only if there are $N$ disjoint sections to $\pi$, i.e. continuous maps $\tau_{i}: X \rightarrow Y, 1 \leq i \leq N$, with disjoint images, satisfying $\left(\pi \tau_{i}\right)(x)=x$ for all $x$ in $X$.

(3) An $N$-point extension of $(X, \sigma)$ is $\operatorname{SFT}$ if and only if $(X, \sigma)$ is SFT.

Example 6.3. A constant $N$-to-1 factor map of SFTs need not be an $N$-point extension; for example, the matrix $\widetilde{C}$ below is the adjacency matrix of a labeled graph for which the labels define a one-block code of edge SFTs, $X_{C} \rightarrow X_{\bar{C}}$, which is constant 2-to-1 but is not a 2-point extension.

$$
\widetilde{C}=\left(\begin{array}{cccc}
a & 0 & b & 0 \\
0 & a & b & 0 \\
0 & 0 & c & 0 \\
0 & 0 & 0 & c
\end{array}\right) \quad C=\left(\begin{array}{cccc}
1 & 0 & 1 & 0 \\
0 & 1 & 1 & 0 \\
0 & 0 & 1 & 0 \\
0 & 0 & 0 & 1
\end{array}\right) \quad \bar{C}=\left(\begin{array}{ll}
1 & 1 \\
0 & 1
\end{array}\right)
$$

In contrast, we have the following key fact, which follows immediately from Nasu's work [34] (cited in [1, p. 489, Remarks (iv)]) after a translation of terminology. Tools for a proof (not a stated result) can also be found in [28, Sec. 4.3].

Theorem 6.4. [34, Theorem 7.3; see also Corollary 6.6] Suppose $\pi$ is a constant $N-$ to-1 factor map between irreducible SFTs. Then $\pi$ is an $N$-point extension.

We define two $N$-point extensions to be isomorphic if they are isomorphic as factor maps. The following standard fact is another routine exercise.

Fact 6.5. $N$-point extensions, defined by data $(X, \sigma, \tau)$ and $\left(X^{\prime}, \sigma^{\prime}, \tau^{\prime}\right)$ as in Definition 6.1, with $\tau$ and $\tau^{\prime}$ skewing from the left, are isomorphic if and only if there is a conjugacy $\varphi:(X, \sigma) \rightarrow\left(X^{\prime}, \sigma^{\prime}\right)$ such that $\tau$ and $\left(\tau^{\prime} \varphi\right)$ are cohomologous in $(X, \sigma)$ : i.e., there is a continuous $\gamma: X \rightarrow \mathcal{S}_{N}$ such that for all $x$ in $X$,

$$
\tau^{\prime}(\varphi(x))=\gamma(\sigma(x)) \tau(x)(\gamma(x))^{-1}
$$

(where the right hand side is a product in the group $\mathcal{S}_{N}$ ).

Definition 6.6. In this paper, a $G$-SFT is a shift of finite type $X$ together with a free, continuous shift-commuting action of a finite group $G$. The factor map of the $G$-SFT is the everywhere $|G|$-to-1 map which collapses $G$-orbits to points. The $G$-SFT is a left $G$-SFT if the $G$-action is a left action $(g: x \mapsto g x ; g h: x \mapsto g(h x))$; it is a right $G$-SFT if the $G$-action is a right action $(g: x \mapsto x g ; g h: x \mapsto(x g) h)$. 
By definition, a conjugacy (isomorphism) of two G-SFTs is a topological conjugacy of the underlying SFTs which intertwines their $G$ actions; equivalently, it is a conjugacy of the factor maps of the $G$-SFTs which intertwines their $G$-actions.

The factor map of a right $G$-SFT is a $|G|$-point extension, and therefore can be presented as in Definition 6.1, with $G$ in place of the set $\{1, \ldots, N\}$. Here the permutation $\tau_{x}$ of Facts 6.2 must be left multiplication by some element $\beta(x)=\beta_{x}$ of $G$, as the right $G$-action commuting with the shift forces for $g$ in $G$ that $\tau_{x}(e g)=\tau_{x}(e) g$.

Fact 6.7. Two right $G$-SFTs $(X, \sigma)$ and $\left(X^{\prime}, \sigma^{\prime}\right)$ with right $G$-actions $\beta$ and $\beta^{\prime}$ are isomorphic if and only if there is a conjugacy $\varphi: X \rightarrow X^{\prime}$ and a continuous $c: X \rightarrow G$ such that for all $x$ in $X$,

$$
\beta^{\prime}(\varphi(x))=c(\sigma(x)) \beta(x) c(x)^{-1} .
$$

(where the right hand side is the product in the group $G$ ).

Fact 6.7 holds because the permutation $\gamma(x)$ in Fact 6.5 is here an element of $\mathcal{S}_{G}$ which commutes with the right $G$-action, and again must be left multiplication by some element $c(x)$ of $G$.

As noted in [1]: every 2-point extension of SFTs is isomorphic to the factor map of some $G$-SFT with $G=\mathbb{Z} / 2 \mathbb{Z}$ (Remark 6.10 gives one proof), but for $N>2$, an $N$-point extension of an SFT is not in general isomorphic to the factor map of a $G$-SFT. For example, if $\pi:(Y, \rho) \rightarrow(X, \sigma)$ is the factor map of a $G$-SFT, then two $\rho$-periodic points with the same image must have the same $\rho$-period; but a 3 -point extension could collapse a fixed point and an orbit of size 2 to a fixed point.

Nevertheless, the classification of $N$-point extensions of SFTs can be reduced to the classification of $G$-SFTs.

Definition 6.8. [1, p. 493] The full extension of an $N$-point extension of a system $(X, \sigma)$, presented as above by $\tau: X \rightarrow \mathcal{S}_{N}$, is the self map of $X \times \mathcal{S}_{N}$ defined by the rule $(x, \alpha) \mapsto(\sigma(x), \tau(x) \alpha)$, (here $\tau(x) \alpha$ is the product in $\left.\mathcal{S}_{N}\right)$ with right $\mathcal{S}_{N^{-}}$action $h:(x, g) \mapsto(x, g h)$.

Proposition 6.9. Let $\pi:(Y, \rho) \rightarrow(X, \sigma)$ and $\pi^{\prime}:\left(Y^{\prime}, \rho^{\prime}\right) \rightarrow\left(X^{\prime}, \sigma^{\prime}\right)$ be $N$-point extensions of two shifts of finite type $X$ and $X^{\prime}$, presented by skewing functions $\tau, \tau^{\prime}$ acting from the left as in Definition 6.1. Then the following are equivalent.

(1) $\pi$ and $\pi^{\prime}$ are conjugate factor maps.

(2) There is a conjugacy $\varphi:(X, \sigma) \rightarrow\left(X^{\prime}, \sigma^{\prime}\right)$ and a continuous $\gamma: X \rightarrow \mathcal{S}_{N}$ such that for all $x$ in $X$,

$$
\tau^{\prime}(\varphi(x))=\gamma(\sigma(x)) \tau(x)(\gamma(x))^{-1} .
$$

(3) The associated full extensions are conjugate right $\mathcal{S}_{N}$-SFTs.

Proof. (1) $\Longleftrightarrow(2)$ is Fact 6.5, and (3) $\Longleftrightarrow(2)$ is Fact 6.7 , 
In [1, Theorem 4.2(B,C)], Adler, Kitchens and Marcus provided easily computed group invariants for almost topological conjugacy, and hence conjugacy, of certain full extensions (the nonwandering $\mathcal{S}_{N}$-transitive extensions). (For examples of their use to distinguish $N$-point extensions, see [40, p. 258]). More difficult algebraic invariants are required for a classification of $\mathcal{S}_{N}$-extensions up to conjugacy, or up to flow equivalence [9].

Remark 6.10. [1, p. 494] Let a full extension $\tilde{\pi}: X \times \mathcal{S}_{N} \rightarrow X$ of an $N$ point extension $\pi: X \times\{1, \ldots, N\}$ be given using $\tau$ as in Definition 6.8. Then the map $\beta: X \times \mathcal{S}_{N} \rightarrow X \times\{1, \ldots, N\}$ given by $(x, g) \mapsto(x, g(1))$ is an $(N-1)$ !-point extension and $\widetilde{\pi}=\pi \beta$. The map $\beta$ is isomorphic to the map obtained by using in place of $g(1)$ the coset $g H$, where $H=\left\{h \in \mathcal{S}_{N}: h(1)=1\right\}$.

Remark 6.11. There are some differences between our presentation and terminology and what's in [1, 2]. We have only taken some of their beginning content - the papers were concerned with almost topological conjugacies of factor maps. Some of our statements are only implicit in [1, 2]. The seminal measurable version of Proposition 6.9 is explicit in Rudolph's paper [40, Lemma 1].

More background on $G$-SFTs can be found in [1, 2, 18] and [16, Appendix A]. The chosen action in [18] should be a left rather than right action, as explained in [16, Appendix A].

\section{PET SOFIC SHIFTS}

In this section, we will use full extensions to reduce the FE classification of a certain class of AFT shifts to the flow equivalence classification of $G$-SFTs, for which complete invariants are known [9]. First, we must address a technical point involving left vs. right actions.

A square matrix $A$ over $\mathbb{Z}_{+} G$ presents a $G$-SFT (equivalently, a $G$ extension of an SFT) in a natural way: the matrix $A$ gives a labeling of edges of a directed graph by elements of $G$, say $e \mapsto \ell(e)$. The graph defines the usual edge SFT and the labeling defines a skewing function $\tau_{x}$ : an element of $G$ is multiplied by $\ell\left(x_{0}\right)$. This is a left $G$ SFT if $\tau_{x}: g \mapsto g \ell\left(x_{0}\right)$. The left $G$-SFTs presented by $A$ and $B$ are topologically conjugate if and only if the matrices $A$ and $B$ are strong shift equivalent over $\mathbb{Z}_{+} G$ (see [18] and [16, Appendix A]). This leads us to a natural definition.

Definition 7.1. The left full extension of an $N$-point extension is defined as in Definition 6.8, with the following changes: $\tau$ is chosen to act from the right, and then $\mathcal{S}_{N}$ is taken to act from the left. (So, the left full extension of an $N$-point extension of an SFT is a left $G$-SFT, with $G=\mathcal{S}_{N}$.) 
Fact 6.2(2) does not distinguish between left and right; the same class of extensions (up to topological conjugacy) is presented with functions skewing from the right as for functions skewing from the left. Arguments for left $G$-SFTs mimicking those for right $G$-SFTs in the last section then lead to the following analogue of Proposition 6.9, with an additional condition (4).

Proposition 7.2. Let $\pi:(Y, \rho) \rightarrow(X, \sigma)$ and $\pi^{\prime}:\left(Y^{\prime}, \rho^{\prime}\right) \rightarrow\left(X^{\prime}, \sigma^{\prime}\right)$ be $N$-point extensions of two shifts of finite type $X$ and $X^{\prime}$, presented by skewing functions $\tau, \tau^{\prime}$ as in Definition 6.1, but with $\tau$ and $\tau^{\prime}$ skewing from the right. Then the following are equivalent.

(1) $\pi$ and $\pi^{\prime}$ are conjugate factor maps.

(2) There is a conjugacy $\varphi:(X, \sigma) \rightarrow\left(X^{\prime}, \sigma^{\prime}\right)$ and a continuous $\gamma: X \rightarrow \mathcal{S}_{N}$ such that for all $x$ in $X$,

$$
\tau^{\prime}(\varphi(x))=(\gamma(x))^{-1} \tau(x) \gamma(\sigma(x)) .
$$

(3) The associated full extensions are conjugate left $\mathcal{S}_{N}$-SFTs.

(4) If the left $\mathcal{S}_{N}$-SFT full extensions are presented by matrices $A, B$ over $\mathbb{Z}_{+} \mathcal{S}_{N}$, then $A$ and $B$ are strong shift equivalent over $\mathbb{Z}_{+} \mathcal{S}_{N}$

If one remains with a presentation of an $N$-point presentation with $\tau$ skewing from the left, one can still reduce to a SSE- $\mathbb{Z}_{+}$invariant. Let $A$ and $B$ be $m \times m$ matrices over $\mathbb{Z}_{+} G$ presenting full extensions for $N$-point presentations $\pi, \pi^{\prime}$ with $\tau$ skewing from the left. As in [16, Appendix A], let $A^{\text {opp }}$ to be the $m \times m$ matrix such that $A(i, j)=$ $\sum_{g} n_{g} g \Longrightarrow A^{\mathrm{opp}}(i, j)=\sum_{g} n_{g} g^{-1}$, and likewise define $B^{\mathrm{opp}}$. Then $\pi$ and $\pi^{\prime}$ will be isomorphic $N$-point extensions if and only if $A^{\text {opp }}$ and $B^{\text {opp }}$ are SSE- $\mathbb{Z}_{+}$(i.e., define isomorphic left $\mathcal{S}_{N^{-}}$SFTs).

The condition (2) in Proposition 7.2 reflects the following analogue of Fact 6.7.

Fact 7.3. Two left $G$-SFTs $(X, \sigma)$ and $\left(X^{\prime}, \sigma^{\prime}\right)$ with left $G$-actions $\beta$ and $\beta^{\prime}$ are isomorphic if and only if there is a conjugacy $\varphi: X \rightarrow X^{\prime}$ and a continuous $c: X \rightarrow G$ such that for all $x$ in $X$,

$$
\beta^{\prime}(\varphi(x))=(c(x))^{-1} \beta(x) c(\sigma(x))
$$

(where the right hand side is the product in the group $G$ ).

It is elementary but important to note that for a nonabelian group $G$, the cohomology equations (6.1) and (7.1) are not equivalent. Here is a simple example (distilled from [16, Example A.4]).

Example 7.4. Let $G$ be nonabelian. Let $a, b$ be group elements such that $a b \neq b a$. Let $d=(a b)^{-1}$; then $a b d=e \neq d b a$. Let $X=$ $\left\{x, \sigma(x), \sigma^{2}(x)\right\}$, a single orbit containing three points. Define $\beta(x)=$ $a, \beta(\sigma(x))=b, \beta\left(\sigma^{2}(x)\right)=d$ and $\beta^{\prime}(x)=\beta^{\prime}(\sigma(x))=\beta^{\prime}\left(\sigma^{2}(x)\right)=e$. Then there is a function $c(x)$ satisfying (6.1), but there is no $c(x)$ satisfying (7.1). 
We now turn to flow equivalence. If $X$ is a left $G$-SFT, then the left $G$-action induces a left $G$-action on SX. Two left $G$-SFTs are $G$ flow equivalent if there exists a flow equivalence $\psi: \mathrm{S} X \rightarrow \mathrm{S} X^{\prime}$ which intertwines the $G$-actions.

Proposition 7.5. Suppose $\pi$ and $\pi^{\prime}$ are $N$-point extensions of SFTs. Then the following are equivalent.

(1) As factor maps, $\pi$ and $\pi^{\prime}$ are flow equivalent.

(2) The left full extensions of $\pi$ and $\pi^{\prime}$ are $\mathcal{S}_{N}$-flow equivalent left $\mathcal{S}_{N}$-SFTs.

Proof. We will first prove the implication $(1) \Longrightarrow(2)$. For this we define symbol expansion for $N$-point extensions. Let $\pi:(Y, \rho) \rightarrow(X, \sigma)$ be an $N$-point extension of a shift space, presented by the skewing function $\tau$ as in Definition 6.1, but with $\tau$ skewing from the right, and with $\pi: Y \rightarrow X$ a one-block code. Let $a \in \mathfrak{a}(X)$, let $\star$ be a symbol which does not belong to $\mathfrak{a}(X)$, and let $\tau_{\star} \in \mathcal{S}_{N}$. Then the $N$-point extension $\mathfrak{e}_{a, \tau_{\star}}(\pi):\left(\mathfrak{e}_{a, \tau_{\star}}(Y), \mathfrak{e}_{a, \tau_{\star}}(\rho)\right) \rightarrow\left(\mathfrak{e}_{a, \tau_{\star}}(X), \mathfrak{e}_{a, \tau_{\star}}(\sigma)\right)$ where $\mathfrak{e}_{a, \tau_{\star}}(X)=$ $\mathfrak{e}_{a}(X)$ (cf. Definition 2.3 and the remark just after the proof of Lemma $2.4), \mathfrak{e}_{a, \tau_{\star}}(\sigma)=\sigma_{\tilde{\mathfrak{a}}}, \mathfrak{e}_{a, \tau_{\star}}(Y)=\mathfrak{e}_{a, \tau_{\star}}(X) \times\{1, \ldots, N\}, \mathfrak{e}_{a, \tau_{\star}}(\rho): \mathfrak{e}_{a, \tau_{\star}}(Y) \rightarrow$ $\mathfrak{e}_{a, \tau_{\star}}(Y)$ is given by $\mathfrak{e}_{a, \tau_{\star}}(\rho)((x, k))=\left(\mathfrak{e}_{a, \tau_{\star}}(\sigma)(x), \mathfrak{e}_{a, \tau_{\star}}(\tau)_{x}(k)\right)$ with $\mathfrak{e}_{a, \tau_{\star}}(\tau)_{x}=\tau_{x^{\prime}}$ if $x=\iota_{a}\left(x^{\prime}\right)$, and $\mathfrak{e}_{a, \tau_{\star}}(\tau)_{x}=\tau_{\star}$ if $x=\sigma_{\tilde{\mathfrak{a}}}\left(\iota_{a}\left(x^{\prime}\right)\right)$, and $\mathfrak{e}_{a, \tau_{\star}}(\pi): \mathfrak{e}_{a, \tau_{\star}}(Y) \rightarrow \mathfrak{e}_{a, \tau_{\star}}(X)$ is given by $\mathfrak{e}_{a, \tau_{\star}}(\pi)(x, k)=x$, is called a symbol expansion of $\pi:(Y, \rho) \rightarrow(X, \sigma)$. An argument similar to the one used in the proof of the Parry-Sullivan Theorem [37] shows that flow equivalence of $N$-point extensions of shift spaces is generated by conjugacy and symbol expansions. It follows from Proposition 6.9 that conjugacy of $N$-point extensions gives conjugacy of the full extensions, and it is easy to check the full extensions of $\pi:(Y, \rho) \rightarrow(X, \sigma)$ and $\mathfrak{e}_{a, \tau_{\star}}(\pi):\left(\mathfrak{e}_{a, \tau_{\star}}(Y), \mathfrak{e}_{a, \tau_{\star}}(\rho)\right) \rightarrow\left(\mathfrak{e}_{a, \tau_{\star}}(X), \mathfrak{e}_{a, \tau_{\star}}(\sigma)\right)$ are flow equivalent. It follows that $(1) \Longrightarrow(2)$.

We will now prove $(2) \Longrightarrow(1)$. If $\pi:(Y, \rho) \rightarrow(X, \sigma)$ is an $N$ point extension, then we can recover $\mathrm{S} Y, \mathrm{~S} X$, and $\mathrm{S} \pi: \mathrm{S} Y \rightarrow \mathrm{S} X$ from $\mathrm{S}\left(X \times \mathcal{S}_{N}\right)$ as in Remark 6.10. It follows that (2) $\Longrightarrow(1)$.

Remark 7.6. For a finite group $G$, every $G$-SFT can be presented by a square matrix over $\mathbb{Z}_{+} G$ as described above. Complete algebraic invariants for $G$-flow equivalence are known, by [18] in the case the extension is mixing and by [9] in general. For further discussion see [9, 16].

Definition 7.7. An irreducible sofic shift is point extension type (PET) if it has a Fischer cover $\pi$ such that for each $k$ in $\operatorname{MultiCard}(\pi)$, the set $\mathrm{M}_{k}(\pi)$ is a closed (and hence a subshift).

In Definition 7.7, it would be equivalent to require each $\mathrm{M}_{k}^{-1}(\pi)$ to be closed (hence a subshift). Note that among irreducible shifts, a PET 
sofic shift must be AFT (by Theorem5.2(vii)), and a near Markov shift must trivially be PET, with both inclusions proper. We will justify the PET name with Lemma 7.9, whose proof appeals to the following result of Jung.

Fact 7.8. 25] A constant $k$-to-1 biclosing factor map between subshifts is a $k$-point extension.

Nasu 34] proved Fact 7.8 in the case the subshifts are irreducible SFTs. The general result is contained in [25, Prop. 4.5].

Lemma 7.9. Suppose $Y$ is an irreducible PET sofic shift with Fisher cover $\pi: X_{A} \rightarrow Y$. Then for each $k$ in $\operatorname{MultiCard}(\pi)$, the restriction $\pi: \mathrm{M}_{k}^{-1}(\pi) \rightarrow \mathrm{M}_{k}(\pi)$ is a k-point extension of a shift of finite type.

Proof. For $k \in \mathbb{N}$ we define

$$
E_{k}(\pi)=E(\pi) \cap\left(\mathrm{M}_{k}^{-1}(\pi) \times \mathrm{M}_{k}^{-1}(\pi)\right),
$$

and let

$$
E(\pi)=\left\{(w, x) \in X_{A} \times X_{A}: \pi(w)=\pi(x)\right\} .
$$

Then $E_{k}(\pi) \neq \emptyset$ only if $k \in \operatorname{MultiCard}(\pi)$ or $k=1$, and $E_{1}(\pi)=$ $\left\{(x, x): x \in X_{A}\right\}$. The set $E(\pi)$ of $\pi$ is a shift of finite type in $X_{A} \times X_{A}$, and equals the disjoint union of the shifts $E_{k}(\pi)$. Therefore each $E_{k}(\pi)$ is also SFT. For $k \in \operatorname{MultiCard}(\pi)$, define $p_{k}: E_{k}(\pi) \rightarrow \mathrm{M}_{k}^{-1}(\pi)$ by $p_{k}:(x, y) \mapsto x$. Then $p_{k}$ is biclosing (because $\pi$ is biclosing), and everywhere $k$-to- 1 . Then by Fact $7.8, p_{k}$ is a $k$-point extension. Because $E_{k}(\pi)$ is SFT, it follows that $\mathrm{M}_{k}^{-1}(\pi)$ is SFT. Then the same argument applied to the restriction $\pi: \mathrm{M}_{k}^{-1}(\pi) \rightarrow \mathrm{M}_{k}(\pi)$ shows this map is a $k$-point extension of a shift of finite type.

Theorem 7.10. A subshift flow equivalent to an irreducible PET sofic shift must also be irreducible PET sofic.

Suppose $X$ and $X^{\prime}$ are irreducible PET sofic shifts with Fisher covers $\pi: X_{A} \rightarrow X$ and $\pi^{\prime}: X_{A^{\prime}} \rightarrow X^{\prime}$. Then the following are equivalent.

(1) The shifts $X$ and $X^{\prime}$ are flow equivalent.

(2) The SFTs $X_{A}, X_{A^{\prime}}$ are flow equivalent;

$$
\operatorname{MultiCard}(\pi)=\operatorname{MultiCard}\left(\pi^{\prime}\right) \text {; }
$$

and for each $k$, the left full extensions of $\left.\pi\right|_{\mathrm{M}_{k}^{-1}(\pi)}$ and $\left.\pi^{\prime}\right|_{\mathrm{M}_{k}^{-1}\left(\pi^{\prime}\right)}$ are $\mathcal{S}_{k}$-flow equivalent left $\mathcal{S}_{k}$-SFTs.

Proof. The invariance of the irreducible sofic PET class under flow equivalence is clear. It follows from Proposition 7.5 that condition (2) is equivalent to the following condition $\left(2^{\prime}\right)$ : the SFTs $X_{A}, X_{A^{\prime}}$ are flow equivalent, and for each $k$, the factor maps $\left.\pi\right|_{\mathrm{M}_{k}^{-1}(\pi)}$ and $\left.\pi^{\prime}\right|_{\mathrm{M}_{k}^{-1}\left(\pi^{\prime}\right)}$ are flow equivalent. By the Reduction Theorem 5.3. we have $(1) \Longleftrightarrow\left(2^{\prime}\right)$. Theorem 7.10 follows. 
We next point out a specific case of Theorem 7.10.

Theorem 7.11. Suppose $Y$ is AFT with Fischer cover $\pi: X \rightarrow Y$ for which $\operatorname{MultiCard}(\pi)$ is a singleton $\{k\}$ (e.g., if $Y$ is $A F T$ and no point of $Y$ has more than 2 preimages under $\pi)$. Then $Y$ is PET and $Y$ is classified up to flow equivalence by the invariants of Theorem 7.10.

Proof. Because $Y$ is $\mathrm{AFT}, \mathrm{M}_{k}^{-1}(\pi)=\mathrm{M}^{-1}(\pi)$ is closed, so $Y$ is PET and is therefore classified up to flow equivalence by the invariants of Theorem 7.10.

Remark 7.12. Assuming the AFT shifts $X$ and $X^{\prime}$ are strictly sofic, the SFTs $X_{A}, X_{A^{\prime}}$ in the statement of Theorem 7.10 must be nontrivial irreducible SFTs (as a strictly sofic irreducible AFT shift has more than one orbit); they are then flow equivalent if and only if $\operatorname{cok}(I-A) \cong$ $\operatorname{cok}\left(I-A^{\prime}\right)$ and $\operatorname{det}(I-A)=\operatorname{det}\left(I-A^{\prime}\right)$ (see [20]). For any finite group $G$, complete algebraic invariants for $G$-SFTs are known (by [18] for mixing extensions and by [9] in general), but are considerably more complicated.

Remark 7.13. Suppose $Y, Y^{\prime}$ are SFTs. Let $X$ be a mixing SFT containing disjoint copies $Y_{1}, Y_{2}$ of $Y$ and also containing disjoint copies $Y_{1}^{\prime}, Y_{2}^{\prime}$ of $Y^{\prime}$. Let $\alpha: Y_{1} \rightarrow Y_{2}$ be a topological conjugacy. Define a sofic shift $T$ as the quotient $\pi: X \rightarrow T$ where $\pi(x)=\pi(\alpha(x))$ if $x \in Y_{\text {! }}$ and $\pi$ identifies no other points. Similarly define $T^{\prime}$ via a conjugacy $\alpha^{\prime}: Y_{1}^{\prime} \rightarrow$ $Y_{2}^{\prime}$. By Theorem 7.10, $T$ is flow equivalent to $T^{\prime}$ if and only if $Y$ is flow equivalent to $Y^{\prime}$. Thus the classification of irreducible sofic shifts up to flow equivalence requires the full classification of general (reducible) SFTs up to flow equivalence. (Indeed, this was one motivation for Huang's original investigation [23].) There is a decision procedure for determining whether two SFTs are flow equivalent [17].

Remark 7.14. If for example $\pi$ is an $N$-point extension between mixing SFTs, then the full extension will be a disjoint union of topologically conjugate irreducible SFTs [1, p. 495]. These are not necessarily $G$-invariant; still, in this case their equivariant flow equivalence classification can be quickly reduced to the flow equivalence classification of G-SFTs which are mixing (see [18, Section 4] or [9, Section 3]).

But for the classification of general PET irreducible sofic shifts, the classification of general reducible $G$-SFTs is required. Indeed, suppose $G$ is a finite group and two $N$-point extensions are the quotient maps of $G$-actions; then the $N$-point extensions are topologically conjugate if and only if the $G$-actions are topologically conjugate (there is no need to introduce the full extension). For every $G$-SFT, its quotient map can be realized as the restriction of a Fischer cover of an irreducible PET sofic shift to its multiplicity shift. Thus the problem of classifying sofic shifts up to conjugacy (or flow equivalence) contains the problem of classifying $G$-SFTs. 
Remark 7.15. For irreducible sofic shifts, let's note how the PET condition is a natural refinement of the AFT condition. Suppose $\pi: X_{A} \rightarrow Y$ is a Fischer cover. Define (recalling Definition 3.4 and (7.2), (7.3)

$$
\begin{aligned}
& \widetilde{\mathrm{M}}_{\leq k}(\pi)=\bigcup_{j \leq k} E_{j}(\pi) \\
& \mathrm{M}_{\geq k}^{-1}(\pi)=\bigcup_{j \geq k} \mathrm{M}_{j}^{-1}(\pi) .
\end{aligned}
$$

Then $Y$ is AFT if and only if $\widetilde{\mathrm{M}}_{<1}(\pi)$ is isolated in $E(\pi) ; Y$ is PET if and only if for each $k, \widetilde{\mathrm{M}}_{\leq k}(\pi)$ is isolated in $E(\pi)$. Similarly, $Y$ is AFT if and only if $\mathrm{M}_{\geq 2}^{-1}(\pi)$ is closed; $Y$ is PET if and only if $\mathrm{M}_{\geq k}^{-1}(\pi)$ is closed for each $k \geq 2$.

Remark 7.16. Given a Fischer cover $\pi: X \rightarrow Y$. Define $\widetilde{\pi}: E(\pi) \rightarrow$ $\mathrm{M}^{-1}(\pi)$ by $(x, w) \mapsto x$. Then the following are equivalent.

(1) $Y$ is PET.

(2) On each indecomposable component of $E(\pi), \widetilde{\pi}$ is constant-toone; and if $C, C^{\prime}$ are indecomposable components $E(\pi)$ with $\tilde{\pi}(C) \cap \widetilde{\pi}\left(C^{\prime}\right) \neq \emptyset$, then $\widetilde{\pi}(C)=\widetilde{\pi}\left(C^{\prime}\right)$.

\section{AlgORIThMS FOR PET SOFIC ShifTS}

In this section, we briefly address decision procedures, computations and range of invariants for irreducible PET sofic shifts. Throughout, $\pi: X_{B} \rightarrow Y$ is a given right Fischer cover of an irreducible sofic shift $Y$. This cover is presented by a graph $G_{B}$, with edges of $G_{B}$ labeled by elements of $\mathfrak{a}(Y)$, the alphabet of $Y$, according to the 1-block code $\pi$.

\section{Deciding whether $Y$ is PET.}

To begin, we describe a variant of the subset construction. Enumerate the vertices of the graph $G_{B}$ given by $B$ as $\{1, \ldots, n\}$ and for $1 \leq k \leq n$, let $\mathcal{T}_{k}$ denote the set of ordered tuples consisting of $k$ distinct elements drawn from $\{1, \ldots, k\}$, with elements written in increasing order. We denote an element $i$ of $\mathcal{T}_{k}$ by $i_{1} i_{2} \cdots i_{k}$, with $i_{1}<i_{2}<\cdots<i_{k}$, and set $\mathcal{T}=\bigcup_{1 \leq k \leq n} \mathcal{T}_{k}$. For $i=i_{1} i_{2} \cdots i_{k}$, we also define $|i|=k$ and $\{i\}=\left\{i_{1}, \ldots, i_{k}\right\}$, and define $f(i, a)$ to be the set of terminal vertices of $G_{B}$ edges which have label $a$ and which have initial vertex in $\{i\}$.

From the labeled graph $G_{B}$ we will recursively construct vertex sets $\mathcal{V}(m)$ with the aim of defining an $\mathfrak{a}(Y)$-labeled graph. $\mathcal{V}(0)$ is the singleton $\{i\}$ such that $|i|=n$, and given $\mathcal{V}(m)$, we define

$$
\begin{gathered}
\mathcal{V}(m+1)= \\
\mathcal{V}(m) \cup\{j \in \mathcal{T}: \exists a \in \mathfrak{a}(Y), i \in \mathcal{V}(m) \text { such that } f(i, a)=\{j\}\} .
\end{gathered}
$$


Take $M$ such that $\mathcal{V}(M)=\mathcal{V}(M+1)$. We equip $\mathcal{V}(M)$ with an edge set $\mathcal{E}(M)$ as follows. For $i=i_{1} \cdots i_{k}$ and $j=j_{1} \cdots j_{\ell}$ in $\mathcal{V}(M)$, there is an edge from $i$ to $j$ labeled $a$ if and only if the following hold:

(1) $\{j\}=f(i, a)$, and

(2) if $k>\ell=1$, then at least two edges with initial vertex in $\{i\}$ have label $a$.

Now let $\bar{G}(\pi)$ be the maximum labeled subgraph of $G(M)=(\mathcal{V}(M), \mathcal{E}(M))$ such that every vertex has an incoming edge and an outgoing edge. Let $\overline{\mathcal{V}}_{k}$ be the set of vertices $i$ of $\bar{G}(\pi)$ such that $|i|=k$. Let $\bar{G}_{k}(\pi)$ be the labeled subgraph of $\bar{G}(\pi)$ with vertex set $\overline{\mathcal{V}}_{k}$. Note, because $\pi$ is right resolving, if there is an edge in $\bar{G}(\pi)$ from $i$ to $j$, then $|i| \geq|j|$.

Let $\bar{B}$ be an adjacency matrix for the graph $\bar{G}(\pi)$. Let $\bar{B}_{k}$ be the principal submatrix of $\bar{B}$ on its indices in $\overline{\mathcal{V}}_{k}$. Let $\bar{X}$ denote $X_{\bar{B}}$ and let $\bar{X}_{k}$ be the subshift of $\bar{X}$ which is the edge shift defined from the subgraph of $\bar{G}(\pi)$ with vertex set $\overline{\mathcal{V}}_{k}$. Let $\bar{\varphi}: X_{\bar{B}} \rightarrow Y$ be the one-block map given from the edge labeling of $\bar{G}(\pi)$, and let $\bar{\varphi}_{k}$ be the restriction of $\bar{\varphi}$ to $\bar{X}_{k}$. Then $\bar{\varphi}_{k}$ maps $\bar{X}_{k}$ onto the subset of $\mathrm{M}_{k}(\pi)$ whose $k$ preimages are uniformly separated.

Suppose $\bar{G}(\pi)=\bar{G}$ contains an edge $i \rightarrow j$ for which $|i|>|j|$. Then there exist $k>h$ and a biinfinite path $x$ in $\bar{G}$ such that $x_{n}$ is a $\bar{G}_{k}$ edge for all but finitely many negative $n$ and $x_{n}$ is a $\bar{G}_{h}$ edge for all but finitely many positive $n$. If $h=1$, then using condition (2) we conclude $Y$ is not AFT, hence not PET. If $h>1$ and $Y$ is AFT, then $\pi$ is biclosing and we have points of $\mathrm{M}_{k}^{-1}(\pi)$ in the closure of $\mathrm{M}_{h}^{-1}(\pi)$, and $\pi$ is not PET.

We summarize with the following proposition.

Proposition 8.1. For a Fischer cover $\pi: X \rightarrow Y$ for an irreducible $Y$, the following are equivalent.

(1) $Y$ is PET.

(2) $\bar{G}(\pi)=\bigcup_{k} \bar{G}_{k}(\pi)$

(i.e., if e is an edge in $\bar{G}(\pi)$ from $i$ to $j$, then $|i|=|j|$ ).

Examples 8.2. Below are two matrices which present labeled graphs of Fischer covers, $\pi: X \rightarrow Y$.

$$
B=\left(\begin{array}{ccc}
a+f & 0 & c \\
0 & a & b \\
d & b & a
\end{array}\right), \quad C=\left(\begin{array}{cccc}
d & e & 0 & 0 \\
f & a+d & b & c \\
0 & c & a & b \\
0 & b & c & a
\end{array}\right)
$$

(For example $B$ presents a labeling of a graph with adjacency matrix $\left(\begin{array}{lll}2 & 0 & 1 \\ 0 & 1 & 1 \\ 1 & 1 & 1\end{array}\right)$.) For $\pi$ defined by $B$, the sofic shift $Y$ is AFT but not PET; the subshift $\mathrm{M}_{2}^{-1}(\pi)$ is not closed, and in $\bar{G}(\pi)$ we see the edge

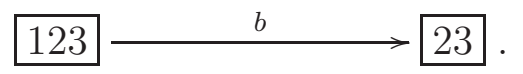


For $Y$ defined by $C, Y$ is PET; the vertices of $\bar{G}(\pi)$ are 234 and 12 , and there is no edge between these. (Here, 234 and 12 have in common the vertex entry 2. In general, for $k>\ell \geq 2$, if $i \in \mathcal{V}_{k}$ and $j \in \mathcal{V}_{\ell}$ and $i, j$ have more than one vertex entry in common, then $Y$ is not PET.)

Remark 8.3. Note that thus far we might as well have indexed the vertices of $\bar{G}(\pi)$ by sets rather than ordered tuples, as in [4]. As we will see below, the ordering becomes necessary for reading off the skewing functions.

\section{Computing $\mathbb{Z}_{+} G$ matrices for PET systems.}

Suppose that $Y$ is PET, with right Fischer cover $\pi: X_{B} \rightarrow Y$ and with $\bar{\pi}: \bar{X} \rightarrow Y$ constructed as above. Suppose $k \in \operatorname{MultiCard}(\pi)$.

We define a labeling of the edges of the graph $\bar{G}_{k}$ as follows. Given such an edge $E$, from vertex $i$ to vertex $j$ in $\overline{\mathcal{V}}_{k}$, we let $\tau(E)$ be the unique permutation $\tau$ of $\{1, \ldots, k\}$ such that for $1 \leq t \leq k$ there is an edge labeled $a$ from $i_{t}$ to $j_{\tau(t)}$. The skewing function defined by $x \mapsto$ $\tau\left(x_{0}\right)$ defines a $k$ point extension $\left(\bar{X}_{k} \times\{1, \ldots, k\}, \bar{\rho}_{k}\right)$ of $\left(\bar{X}_{k}, \sigma\right)$. The square matrix $B_{k}$ over $\mathbb{Z}_{+} \mathcal{S}_{k}$ which presents this extension is defined as follows: $B_{k}(i, j)=\sum_{E} \tau(E)$, where the sum is over the edges $E$ from $i$ to $j$ in $\bar{G}_{k}$. The matrix $\bar{B}_{k}$ defined earlier is indeed the image of $B_{k}$ under the entrywise augmentation map, $\sum_{g \in \mathcal{S}_{k}} n_{g} g \mapsto \sum_{g} n_{g}$.

Let $\left\{\tau_{j}: 1 \leq j \leq k\right\}$ be the collection of continuous sections such that $\mathrm{M}^{-1}(\pi)$ is the disjoint union of the $k$ sets $\tau_{j}\left(\mathrm{M}_{k}(\pi)\right)$. Explicitly, a point $y$ in $\mathrm{M}_{k}(\pi)$ has $k$ preimages, whose zero coordinate symbols $x_{0}$ are edges with initial vertices $i_{1}<i_{2}<\cdots<i_{k}$; we choose $\tau_{j}(y)$ to be the $x$ such that $x_{0}$ has initial vertex $i_{j}$. Then there is a commuting diagram

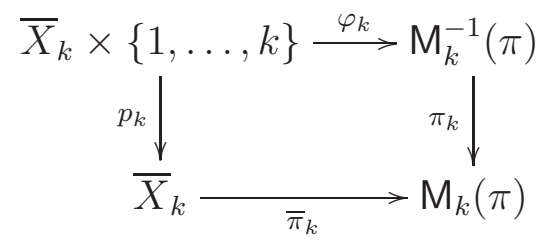

in which $p_{k}:(x, j) \mapsto x ; \bar{\varphi}$ is defined by the edge labeling of $\bar{G}_{k}$; and $\varphi:(x, j) \mapsto \tau_{j}\left(\bar{\varphi}_{k}(x)\right)$. The maps $\varphi_{k}$ and $\bar{\varphi}_{k}$ are homeomorphisms, and $\pi_{k} \varphi_{k}=\bar{\varphi}_{k} p_{k}$. Thus the $k$ point extensions given by $p_{k}$ and $\pi_{k}$ are topologically conjugate.

By Proposition 7.2 , the SSE- $\mathbb{Z}_{+} S_{k}$ class of the matrix $B_{k}$ is a complete invariant for the conjugacy class of the extension $\pi_{k}$. The list of SSE- $\mathbb{Z}_{+} S_{k}$ classes ( $k \in$ MultiCard $(\pi)$ ) is therefore a complete invariant for the restriction of $\pi$ to $M^{-1}(\pi)$. By the extension result [14, Theorem 1.5], this list, together with the SSE- $\mathbb{Z}_{+}$class of the matrix $B$ defining the mixing SFT which is the domain of $\pi$, determines the conjugacy class of $\pi$ up to finitely many possibilities. 
By Theorem 7.10, the classification of PET sofic shifts up to flow equivalence is reduced to the (known) classification of irreducible SFTs up to flow equivalence and the classification of $G$-SFTs up to flow equivalence. Complete algebraic invariants for this are given in 9.

\section{Range of invariants.}

First, suppose $X$ is a mixing SFT, $\mathcal{K}$ is a finite set of positive integers from $[2, \infty)$ and for $k \in \mathcal{K}$ we are given a $k$-point extension of SFTs, $\psi_{k}: U_{k} \rightarrow V_{k}$. Let $\psi$ be the disjoint union of the $\psi_{k}$, with domain the disjoint union $U$ of the $U_{k}$.

Then the following are equivalent.

(1) There is a PET shift $Y$ with Fischer cover $\pi$ whose domain is topologically conjugate to $X$ and whose restriction to $\mathrm{M}^{-1}(\pi)$ is conjugate to $\psi$.

(2) The subshift $U$ embeds into $X$.

Necessary and sufficient conditions for $U$ embedding into $X$ are given by entropy and periodic point counts according to Krieger's Embedding Theorem. So, the embedding constraint is the only constraint for realizing invariants.

Now consider whether $\psi$ can be realized in a specified flow equivalence class of nontrivial mixing SFTs. Trivially, it can, because every such class is contains SFTs defined by arbitrarily large matrices with arbitrarily large entries.

\section{REFERENCES}

[1] R. Adler, B. Kitchens, and B. Marcus. Almost topological classification of finite-to-one factor maps between shifts of finite type. Ergodic Theory Dynam. Systems, 5(4):485-500, 1985.

[2] R. L. Adler, B. Kitchens, and B. H. Marcus. Finite group actions on shifts of finite type. Ergodic Theory Dynam. Systems, 5(1):1-25, 1985.

[3] M. Barge and B. Diamond. A complete invariant for the topology of onedimensional substitution tiling spaces. Ergodic Theory Dynam. Systems, 21(5):1333-1358, 2001.

[4] J. Berstel, C. De Felice, D. Perrin, C. Reutenauer, and G. Rindone. Recent results on syntactic groups of prefix codes. European J. Combin., 33(7):13861401, 2012.

[5] R. Bowen and J. Franks. Homology for zero-dimensional nonwandering sets. Ann. of Math. (2), 106(1):73-92, 1977.

[6] M. Boyle. Lower entropy factors of sofic systems. Ergodic Theory Dynam. Systems, 3(4):541-557, 1983.

[7] M. Boyle. Flow equivalence of shifts of finite type via positive factorizations. Pacific J. Math., 204(2):273-317, 2002.

[8] M. Boyle, T. Carlsen, and S. Eilers. Flow equivalence and isotopy for subshifts. arXiv:1511.03478, November 2015.

[9] M. Boyle, T.M. Carlsen, and S. Eilers. Flow equivalence of G-SFTs. arXiv preprint, November 2015.

[10] M. Boyle and D. Handelman. The spectra of nonnegative matrices via symbolic dynamics. Ann. of Math. (2), 133(2):249-316, 1991. 
[11] M. Boyle and D. Huang. Poset block equivalence of integral matrices. Trans. Amer. Math. Soc., 355(10):3861-3886 (electronic), 2003.

[12] M. Boyle, B. Kitchens, and B. Marcus. A note on minimal covers for sofic systems. Proc. Amer. Math. Soc., 95(3):403-411, 1985.

[13] M. Boyle and W. Krieger. Almost Markov and shift equivalent sofic systems. In Dynamical systems (College Park, MD, 1986-87), volume 1342 of Lecture Notes in Math., pages 33-93. Springer, Berlin, 1988.

[14] M. Boyle and W. Krieger. Automorphisms and subsystems of the shift. J. Reine Angew. Math., 437:13-28, 1993.

[15] M. Boyle, D. Lind, and D. Rudolph. The automorphism group of a shift of finite type. Trans. Amer. Math. Soc., 306(1):71-114, 1988.

[16] M. Boyle and S. Schmieding. Finite group extensions of shifts of finite type: $K$-theory, Parry and Livšic. arXiv:1503.02050, to appear in Ergodic Theory Dynam. Systems, 2015.

[17] M. Boyle and B. Steinberg. Decidability of matrix equivalence and diagram isomorphism, for Cuntz-Krieger $C^{*}$-algebras and flow equivalence (working title). In preparation, 2015.

[18] M. Boyle and M.C. Sullivan. Equivariant flow equivalence for shifts of finite type, by matrix equivalence over group rings. Proc. London Math. Soc. (3), 91(1):184-214, 2005.

[19] A. Costa and B. Steinberg. Flow equivalence of $R$-graph shifts. arXiv:1304.3487, to appear in Ergodic Theory Dynam. Systems, 2014.

[20] J. Franks. Flow equivalence of subshifts of finite type. Ergodic Theory Dynam. Systems, 4(1):53-66, 1984.

[21] M. Fujiwara and M. Osikawa. Sofic systems and flow equivalence. Math. Rep. Kyushu Univ., 16(1):17-27, 1987.

[22] T. Hamachi and M. Nasu. Topological conjugacy for 1-block factor maps of subshifts and sofic covers. In Dynamical systems (College Park, MD, 1986-87), volume 1342 of Lecture Notes in Math., pages 251-260. Springer, Berlin, 1988.

[23] D. Huang. Flow equivalence of reducible shifts of finite type. Ergodic Theory Dynam. Systems, 14(4):695-720, 1994.

[24] R. Johansen. Flow equivalence of sofic beta-shifts. To appear, Ergodic Theory Dynam. Systems.

[25] U. Jung. Open maps between shift spaces. Ergodic Theory Dynam. Systems, 29(4):1257-1272, 2009.

[26] A. Katok and B. Hasselblatt. Introduction to the modern theory of dynamical systems, volume 54 of Encyclopedia of Mathematics and its Applications. Cambridge University Press, Cambridge, 1995. With a supplementary chapter by Katok and Leonardo Mendoza.

[27] K. H. Kim and F. W. Roush. An algorithm for sofic shift equivalence. Ergodic Theory Dynam. Systems, 10(2):381-393, 1990.

[28] B.P. Kitchens. Symbolic dynamics; One-sided, two-sided and countable state Markov shifts. Springer-Verlag, Berlin, 1998.

[29] W. Krieger. On sofic systems. I. Israel J. Math., 48(4):305-330, 1984.

[30] W. Krieger. On flow equivalence of $R$-graph shifts. arXiv:1406.6283, 2014.

[31] D. Lind and B. Marcus. An introduction to symbolic dynamics and coding. Cambridge University Press, Cambridge, 1995.

[32] B. Marcus. Sofic systems and encoding data. IEEE Trans. Inform. Theory, 31(3):366-377, 1985.

[33] K. Matsumoto. $C^{*}$-algebras associated with lambda-synchronizing subshifts and flow equivalence. J. Aust. Math. Soc., 95(2):241-265, 2013. 
[34] M. Nasu. Constant-to-one and onto global maps of homomorphisms between strongly connected graphs. Ergodic Theory Dynam. Systems, 3(3):387-413, 1983.

[35] M. Nasu. Topological conjugacy for sofic systems and extensions of automorphisms of finite subsystems of topological Markov shifts. In Dynamical systems (College Park, MD, 1986-87), volume 1342 of Lecture Notes in Math., pages 564-607. Springer, Berlin, 1988.

[36] V. V. Nemytskii and V. V. Stepanov. Qualitative theory of differential equations. Princeton Mathematical Series, No. 22. Princeton University Press, Princeton, N.J., 1960.

[37] W. Parry and D. Sullivan. A topological invariant of flows on 1-dimensional spaces. Topology, 14(4):297-299, 1975.

[38] G. Restorff. Classification of Cuntz-Krieger algebras up to stable isomorphism. J. Reine Angew. Math., 598:185-210, 2006.

[39] M. Rørdam. Classification of Cuntz-Krieger algebras. K-Theory, 9(1):31-58, 1995.

[40] D.J. Rudolph. Counting the relatively finite factors of a Bernoulli shift. Israel J. Math., 30(3):255-263, 1978.

[41] S. Williams. Covers of non-almost-finite type sofic systems. Proc. Amer. Math. Soc., 104(1):245-252, 1988.

Department of Mathematics, University of Maryland, College PARK, MD 20742-4015, USA

E-mail address: mmb@math.umd.edu

Department of SCIEnCE And TeChnology, University of the FAroe Islands, Nóatún 3, FO-100 Tórshavn, The Faroe Islands

E-mail address: toke.carlsen@gmail.com

Department of Mathematical Sciences, University of CopenHAGen, DK-2100 Copenhagen $\varnothing$, Denmark

E-mail address: eilers@math.ku.dk 